\title{
A!
}

This is an electronic reprint of the original article.

This reprint may differ from the original in pagination and typographic detail.

Mercier de Lepinay, Laure; Damskägg, Erno; Ockeloen-Korppi, Caspar F.; Sillanpää, Mika A.

\section{Realization of Directional Amplification in a Microwave Optomechanical Device}

Published in:

Physical Review Applied

DOI:

10.1103/PhysRevApplied.11.034027

Published: 12/03/2019

Document Version

Publisher's PDF, also known as Version of record

Please cite the original version:

Mercier de Lepinay, L., Damskägg, E., Ockeloen-Korppi, C. F., \& Sillanpää, M. A. (2019). Realization of Directional Amplification in a Microwave Optomechanical Device. Physical Review Applied, 11(3), 1-17. [034027]. https://doi.org/10.1103/PhysRevApplied.11.034027

This material is protected by copyright and other intellectual property rights, and duplication or sale of all or part of any of the repository collections is not permitted, except that material may be duplicated by you for your research use or educational purposes in electronic or print form. You must obtain permission for any other use. Electronic or print copies may not be offered, whether for sale or otherwise to anyone who is not an authorised user. 


\title{
Realization of Directional Amplification in a Microwave Optomechanical Device
}

\author{
Laure Mercier de Lépinay, ${ }^{*}$ Erno Damskägg, Caspar F. Ockeloen-Korppi, and Mika A. Sillanpää \\ Department of Applied Physics, Aalto University, P.O. Box 15100, FI-00076 Aalto, Finland
}

(Received 5 October 2018; revised manuscript received 7 February 2019; published 12 March 2019)

\begin{abstract}
Directional transmission or amplification of microwave signals is indispensable in various applications involving sensitive measurements. In this work we show experimentally how to use a generic cavity optomechanical setup to nonreciprocally amplify microwave signals above $3 \mathrm{GHz}$ in one direction by $9 \mathrm{~dB}$ and simultaneously attenuate the transmission in the opposite direction by $21 \mathrm{~dB}$. We use a device including two on-chip superconducting resonators and two metallic drumhead mechanical oscillators. Application of four microwave pump-tone frequencies allows the design of constructive or destructive interference for a signal tone depending on the propagation direction. The device can also be configured as an isolator with lossless nonreciprocal transmission and $18 \mathrm{~dB}$ of isolation.
\end{abstract}

DOI: 10.1103/PhysRevApplied.11.034027

\section{INTRODUCTION}

The measurement of weak electromagnetic signals does not only require proper amplification; it is also essential to protect the typically fragile signal source from disturbances by the measurement system even when the amplification adds very little noise to the measured signal. In a typical situation in superconducting quantuminformation systems working at microwave frequencies, Josephson-junction parametric amplifiers (JPAs) are used as a nearly-quantum-limited read-out technology. Traditional JPA designs are reciprocal devices (i.e., they amplify signals the same way in either direction). Therefore, unless additional precautions are taken, the sample is exposed to an amplified noise propagating backward from the amplifier's output, potentially compromising its quantum properties.

One way to break the symmetry between forward and backward transmission is to divide the signal into two branches with transfer phases chosen such that, once recombined, signals propagating in each direction interfere differently. In the microwave domain, this is standardly used to build isolators and circulators by threading current loops with a magnetic flux. These components are then inserted between the sample and low-noise amplifiers to make the signal transmission nonreciprocal. This design, however, results in bulky devices that are inconvenient in the cryogenic systems needed for deep cooling of superconducting quantum systems. Moreover, they use strong magnetic fields that may perturb sensitive signal sources.

In the optical domain, directional propagation is also desired, and has been implemented in microspheres, microrings, or toroids where counterpropagating optical

\footnotetext{
*laure.mercierdelepinay@aalto.fi
}

modes exhibit different optomechanically induced transparencies or amplifications. Optomechanically induced nonreciprocity [1] led to a variety of realizations of isolators, circulators, and directional amplifiers showing strong amplification in the optical domain [2-5].

Instead of spatially distinct transfer paths, nonreciprocity can be obtained if several simultaneous transfer processes form a fictitious loop: in this case the interference phase is typically governed by phase-controlled drive. These ideas have been used in the context of Josephson-junction nonreciprocal devices [6-11], which do not require the use of isolators after the sample in microwave quantum experiments. Optical nonlinearities [12-17] or time modulation of dielectric constants [18-21] have also been used to build nonreciprocal devices, the interfering processes generally consisting of simultaneous down-conversions and up-conversions. Physically spinning devices have also been proposed and used in sound amplifiers [22] to break time symmetry.

Even more recently, suitably coupled multimode optomechanical systems [23-29] have been investigated to this end. Indeed, the interaction of two optical modes with ancillary mechanical modes also allows one to produce multiple interfering transfer paths as required. This type of system was adapted recently to promote nonreciprocal coupling between mechanical modes instead and demonstrate a new cooling mechanism [30]. The engineering of multiple constructively interfering transduction paths has also been demonstrated to increase the bandwidth of an rf-to-optical transducer [31].

Finally, an interesting option to realize directional amplification is provided by traveling-wave amplifiers, as recently demonstrated with JPAs in the microwave regime $[32,33]$. The latter, however, accept only a limited input power. 
Nonreciprocal transfer between two cavities featuring the input and output ports of an optomechanical device can be obtained by the balancing of their direct coupling with a second transfer path involving one or several mechanical oscillators (MOs), as suggested [24,34] and experimentally realized [25], demonstrating amplification in the optical domain. Xu et al. [35] proposed a symmetrized scheme where the two paths each incorporate one MO, which was used in recently reported implementations of microwave isolators and circulators [36-38]. While these devices show good isolation, they are not intended to produce gain, and suffer from insertion losses, though modest.

Several types of microwave amplifiers based on microwave optomechanical devices have been demonstrated recently [39-42]. The best realizations have achieved very low noise, even below the quantum limit in a phase-sensitive mode [42]. Optomechanical amplifiers, in comparison with JPA designs, have the benefit of much higher power-handling capability, which derives from a lower nonlinearity. All these devices, however, are reciprocal because multimode interfering pathways were not specifically designed. In this work, we demonstrate how one can achieve frequency-converting directional microwave amplification in a microwave optomechanical system. The system consists of two microwave cavity modes coupled indirectly via two MOs. Under appropriate driving, each $\mathrm{MO}$ creates a frequency-converting amplification path between the cavities as was previously demonstrated for a single MO [40]. With use of two MOs, interference between the two paths allows directional amplification of electromagnetic signals, realizing the scheme proposed in Ref. [43]. We further generalize the formalism in Ref. [43] to include internal losses of the cavity modes present in the experiment.

\section{THEORETICAL DESCRIPTION}

\section{A. Basic scheme}

The frequency-converting directional amplifier with the pumping scheme schematically illustrated in Fig. 1(a) consists of two microwave cavity modes acting as input and output ports and two MOs mediating two transfer paths for excitations, as schematized in Fig. 1(b). The mechanical oscillators have frequencies $\Omega_{1}$ and $\Omega_{2}$ and decay rates $\gamma_{1}$ and $\gamma_{2}$. The two cavity modes have frequencies $\omega_{1}$ and $\omega_{2}$, and they couple equally to both mechanical degrees of freedom. The cavities couple to a read-out and excitation line with external decay rates $\kappa_{1}^{e}$ and $\kappa_{2}^{e}$, and they have internal decay rates $\kappa_{1}^{i}$ and $\kappa_{2}^{i}$. The input and output ports of the amplifier are defined as the frequency ranges around the cavity resonance frequencies $\omega_{1}$ and $\omega_{2}$, respectively. The optomechanical Hamiltonian for the system of
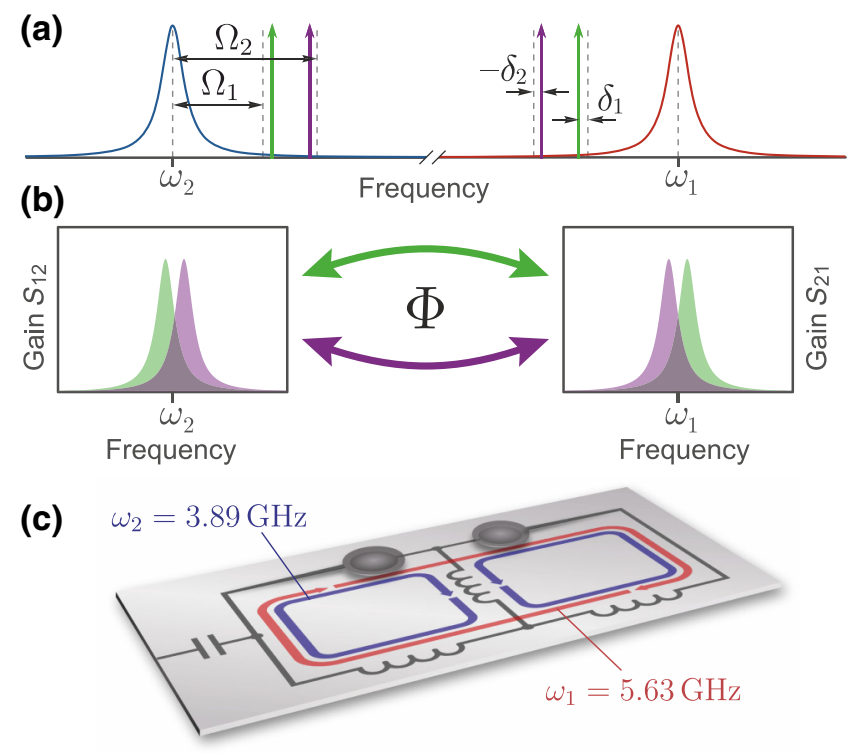

FIG. 1. Implementation of a directional amplifier. (a) Four-tone driving scheme. Two optomechanical cavities, which both couple to two mechanical oscillators, are pumped at frequencies close to either red or blue motional sidebands of the mechanical modes. (b) Two-tone driving of sideband processes corresponding to each mechanical mode [green or purple arrows in (a)] generates bidirectional frequency-converting amplification between cavity modes. Under four-tone driving, interference between the two processes is governed by the relative pump phase $\Phi$, enabling directionality. (c) Schematic representation of the device. A superconducting circuit couples two microwave cavity modes (current flow indicated in blue and red) to two mechanical drumhead resonators. Notice that instead of physically separated ports, the input and output are connected via frequency conversion between the cavity modes.

two cavities and two MOs is

$$
H_{\mathrm{sys}} / \hbar=\sum_{i} \omega_{i} a_{i}^{\dagger} a_{i}+\Omega_{i} b_{i}^{\dagger} b_{i}-\sum_{i j} g_{i j} a_{i}^{\dagger} a_{i}\left(b_{j}+b_{j}^{\dagger}\right),
$$

where we introduced the intracavity field creation and annihilation operators $\left(a_{i}, a_{i}^{\dagger}\right)_{i=1,2}$ and the phononic operators $\left(b_{i}, b_{i}^{\dagger}\right)_{i=1,2}$. The parameters $g_{i j}$ describe the coupling at the single-quantum level. We suppose for the moment that the total linewidths of cavity resonances $\kappa_{j}=\kappa_{j}^{i}+\kappa_{j}^{e}$ are much smaller than the separation of mechanical frequencies, enabling drive tones to independently address each mechanical mode. Each cavity is coupled to each MO through the excitation of one sideband: red sidebands for the input cavity (hereafter named "cavity 1") and blue sidebands for the output cavity (cavity 2), totaling four pump tones as shown in Fig. 1(a). The inclusion of blue-sideband drive tones enables amplification, in contrast to previously demonstrated optomechanical isolators [30,36,37] that used exclusively drive tones close to the red sidebands. 
The device is pumped at frequencies slightly detuned from cavity-1 red sidebands $\left[\omega_{1}-\left(\Omega_{j}-\delta_{j}\right)\right]_{j=1,2}$ and cavity-2 blue sidebands $\left[\omega_{2}+\left(\Omega_{j}-\delta_{j}\right)\right]_{j=1,2}$ [see Fig. 1(a)]. The role of the detunings $\delta_{1}, \delta_{2}$ is discussed later. The pumping enhances and linearizes the coupling, with the resulting multiphoton optomechanical coupling energies given by multiplication of the single-photon coupling with a given field amplitude. These are denoted $G_{i j}\left(J_{i j}\right)$ for the red (blue) sidebands of cavity $i$ and MO $j$. In the proposed pumping scheme, only $G_{1 j}$ and $J_{2 j}$ are nonzero. The cooperativities corresponding to the red and blue sidebands $\left[C_{1 j}=4\left|G_{1 j}\right|^{2} /\left(\gamma_{j} \kappa_{1}\right)\right.$ and $C_{2 j}=$ $4\left|J_{2 j}\right|^{2} /\left(\gamma_{j} \kappa_{2}\right)$, respectively] are similar for both MOs: $C_{11} \simeq C_{12}$ and $C_{21} \simeq C_{22}$, respectively. Furthermore, only one phase $\Phi=\arg \left(G_{11}\right)+\arg \left(J_{21}\right)-\arg \left(G_{12}\right)-\arg \left(J_{22}\right)$ is relevant to the amplifier.

\section{B. Model}

We now recall and adapt the formalism developed in Ref. [43] in particular to include cavity losses. We show that these losses have some experimentally relevant impact that has to be taken into account in a realistic implementation. The evolution equations for photonic operators involve phononic operators and those for phononic operators involve photonic operators. By eliminating phononic operators in the photonic equations of evolution (see Appendix A for more details on the theory), one is left with coupled photonic equations of evolution, with the particularity that the coupling matrix $T$ is not Hermitian. Defining a common vector for intracavity field operators $A \equiv\left(\begin{array}{llll}a_{1} & a_{1}^{\dagger} & a_{2} & a_{2}^{\dagger}\end{array}\right)^{T}$ and corresponding vectors for field input from the external coupler $A_{\text {in }}^{e}$, noise connected to internal losses $A_{\text {in }}^{i}$, and mechanical noise $B_{\text {in }}$, one can define the system susceptibility $\chi(\omega)$ from

$A(\omega)=\chi(\omega)\left[\sqrt{K^{e}} A_{\mathrm{in}}^{e}(\omega)+\sqrt{K^{i}} A_{\mathrm{in}}^{i}(\omega)+U(\omega) B_{\mathrm{in}}(\omega)\right]$,

where $\sqrt{K^{e}} \equiv \operatorname{diag}\left(\sqrt{\kappa_{1}^{e}}, \sqrt{\kappa_{1}^{e}}, \sqrt{\kappa_{2}^{e}}, \sqrt{\kappa_{2}^{e}}\right), \quad \sqrt{K^{i}} \equiv \operatorname{diag}$ $\left(\sqrt{\kappa_{1}^{i}}, \sqrt{\kappa_{1}^{i}}, \sqrt{\kappa_{2}^{i}}, \sqrt{\kappa_{2}^{i}}\right)$, and $U$ is a matrix characterizing the impact of mechanical noise (see Appendix A). The expressions for the spectra providing in particular the added noise and backward-propagating noise are detailed in Appendix B. With this definition, the system susceptibility $\chi$ differs from the susceptibility of two bare, uncoupled cavities $\chi_{c, j}(\omega)=\left(\kappa_{j} / 2-i \omega\right)^{-1}$ by the coupling matrix $T(\omega)$

$$
\chi^{-1}=\operatorname{diag}\left[\chi_{c 1}^{-1},\left(\chi_{c 1}^{*}\right)^{-1}, \chi_{c 2}^{-1},\left(\chi_{c 2}^{*}\right)^{-1}\right]+T,
$$

which is half empty:

$$
T(\omega)=\left(\begin{array}{cccc}
T_{11}(\omega) & 0 & 0 & T_{12}(\omega) \\
0 & T_{11}^{*}(\omega) & T_{12}^{*}(\omega) & 0 \\
0 & T_{21}^{*}(\omega) & T_{22}^{*}(\omega) & 0 \\
T_{21}(\omega) & 0 & 0 & T_{22}(\omega)
\end{array}\right)
$$

where we used the standard convention $\left[T_{i j}(-\omega)\right]^{*}=$ $T_{i j}^{*}(\omega)$. In the following expressions, we took $C_{11}=C_{12}=$ $C_{1}$ and $C_{21}=C_{22}=C_{2}$ though the data are fitted with the general expression, allowing these cooperativities to differ slightly. Each $T_{i j}$ element is the sum of two contributions, one from each MO. In the case of $T_{11}\left(T_{22}\right)$, these contributions represent back-actions on cavity 1 (cavity 2) from driving both MOs, which are added without any multiplying phase factors:

$$
\begin{aligned}
& T_{11}(\omega)=C_{1} \kappa_{1} / 4\left[\gamma_{1} \chi_{m 1}(\omega)+\gamma_{2} \chi_{m 2}(\omega)\right], \\
& T_{22}(\omega)=-C_{2} \kappa_{2} / 4\left[\gamma_{1} \chi_{m 1}(\omega)+\gamma_{2} \chi_{m 2}(\omega)\right],
\end{aligned}
$$

with the mechanical susceptibility $\chi_{m, j}(\omega)=\left[\gamma_{j} / 2\right.$ $\left.-i\left(\omega+\delta_{j}\right)\right]^{-1}$ in the frame defined by the pump frequencies (see the rotating-frame convention in Appendix A). In off-diagonal coefficients on the other hand, each of the two contributions accumulates the phase of two different optomechanical interactions. They are then summed with different phases:

$$
\begin{aligned}
& T_{12}(\omega) \propto\left[e^{i \Phi / 2} \gamma_{1} \chi_{m 1}(\omega)+e^{-i \Phi / 2} \gamma_{2} \chi_{m 2}(\omega)\right], \\
& T_{21}(\omega) \propto-\left[e^{-i \Phi / 2} \gamma_{1} \chi_{m 1}(\omega)+e^{i \Phi / 2} \gamma_{2} \chi_{m 2}(\omega)\right],
\end{aligned}
$$

where the common factor is $\sqrt{C_{1} C_{2} \kappa_{1} \kappa_{2}} / 4$. Further defining the output cavity field $A_{\text {out }}$ analogously to other vectors and using input-output relations $A_{\text {out }}=A_{\text {in }}^{e}-\sqrt{K^{e}} A$, one can get the (optical) transfer matrix $S_{\text {opt }}$ defined by $A_{\text {out }}=$ $S_{\mathrm{opt}} A_{\mathrm{in}}^{e}$ when all noise terms are omitted:

$$
S_{\mathrm{opt}}=\mathbb{I}_{4}-\sqrt{K^{e}} \chi(\omega) \sqrt{K^{e}} .
$$

The expression of the nonzero elements of $S_{\text {opt }}$ in terms of those of $T$ is cumbersome and can be found in Appendix B. However, it is useful to note that $S_{\text {opt }}$ has the same zero elements and symmetries as the coupling matrix $T$. For cavity 1 , each input operator $a_{\mathrm{in}, 1}, a_{\mathrm{in}, 1}^{\dagger}$ then maps to one of the output operators $a_{\mathrm{out}, 2}^{\dagger}, a_{\mathrm{out}, 2}$ of cavity 2 only, which makes the device a phase-insensitive amplifier [44, 45]. Therefore, standard scattering parameters ( $S$ matrix) can be defined as transfer amplitudes involving $a_{1, \text { in }}$ and $a_{2, \text { out }}^{\dagger}: S_{11}=\left.S_{\text {opt }}\right|_{1,1}, S_{22}=\left.S_{\text {opt }}\right|_{4,4}, S_{12}=\left.S_{\text {opt }}\right|_{1,4}$, and $S_{21}=\left.S_{\text {opt }}\right|_{4,1}$. 


\section{Working point in lossy cavities}

The off-diagonal elements of $S$ that characterize backward and forward transfer are proportional to the offdiagonal elements of $T$. Isolation $\left(S_{12}=0\right)$ can therefore be obtained by canceling $T_{12}$ while keeping $T_{21}$ as large as possible, which will make $T$ and $S_{\text {opt }}$ non-Hermitian and $a_{1}$ and $a_{1}^{\dagger}$ eigenvectors of these matrices. However, as can be seen from Eq. (6), this is possible only at $\omega=0$ if the detunings $\delta_{j}$ are nonzero: the two effective mechanical susceptibilities' frequency offset arising from pump detunings is then the only source of directionality. Isolation $S_{12}(0)=$ 0 is furthermore obtained for detunings that compensate the mechanical linewidths' imbalance $\delta_{1}=\gamma_{1} \delta, \delta_{2}=$ $-\gamma_{2} \delta$, and for the phase $\Phi=\arg [(-1+2 i \delta) /(1+2 i \delta)]$. The isolation quality does not depend on cavity losses, and the interference can appear at a different frequency if the detunings do not exactly compensate for the different mechanical linewidths.

One degree of freedom $\delta$ on the detunings is left: it is generally $[36,37]$ tuned to achieve impedance matching of the amplifier to the input line $S_{11}(0)=0$. With nonzero internal cavity losses, this happens when $\delta=$ $\frac{1}{2} \sqrt{2 /\left(2 r_{1}-1\right) C_{1}-1}$, where we introduced $r_{j}=\kappa_{j}^{e} / \kappa_{j}$. This can, however, be realized only for $C_{1}>\left(2 r_{1}-1\right) / 2$ and if $r_{1}>0.5$ (i.e., if the input cavity is not undercoupled). In our experimental case the lower-frequency cavity is undercoupled and is therefore deliberately used as the output cavity.

When isolation and impedance-matching conditions are satisfied (provided this is possible), the forward power gain of the amplifier is

$$
\left|S_{21}(0)\right|^{2}=\frac{r_{2}}{r_{1}} \frac{2 C_{2}\left(2 C_{1}+1-2 r_{1}\right)}{\left[C_{1} /\left(2 r_{1}-1\right)-C_{2}\right]^{2}} .
$$

As previously observed for the single-MO amplifier, the cooperativities are best chosen both large and nearly equal while maintaining $C_{2}\left(2 r_{1}-1\right)<C_{1}$ to prevent parametric instability. This expression coincides with the gain calculated in Ref. [43] in the limit of nonlossy cavities. However, $C_{1}$ now compares to a reduced cooperativity $\left(2 r_{1}-\right.$ 1) $C_{2}$ in the denominator. The power gain for equal and large cooperativities $C_{1}=C_{2}$ is $2 r_{2}\left(2 r_{1}-1\right)^{2} / r_{1}\left(1-r_{1}\right)$. Output-cavity losses (low $r_{2}$ ) are less detrimental to the gain than input-cavity losses, which is a second reason for use of our undercoupled cavity on the output side. A moredetailed analysis of the impact of cavity losses on gain is given in Appendix B. While the impedance-matching condition is required to realize an ideal quantum-limited amplifier, it restricts the choice of operating parameters, and in a practical device a better trade-off may be possible. For example, for our experimental parameters, the maximum gain while strictly enforcing impedance matching and perfect isolation conditions would be $-2.6 \mathrm{~dB}$. By relaxing the impedance-matching condition, we can nevertheless realize directional amplification. We note that impedance mismatch also reduces backward-propagating added noise of the amplifier [43].

\section{EXPERIMENTAL IMPLEMENTATION}

\section{A. Experimental details}

Our device is fabricated by patterning a microwave circuit and mechanical oscillators in aluminum on a quartz substrate. The mechanical elements are circular membranes evaporated on top of a sacrificial amorphous-silicon layer, which is then removed by isotropic reactive-plasma etching to release the membranes. The two drumheads of diameters 19.7 and $16.9 \mu \mathrm{m}$ vibrate above circular electrodes to form displacement-dependent capacitors [46] with fundamental frequencies $\Omega_{1} / 2 \pi=9.24 \mathrm{MHz}$ and $\Omega_{2} / 2 \pi=9.82 \mathrm{MHz}$ and decay rates $\gamma_{1} / 2 \pi \simeq 310 \mathrm{~Hz}$ and $\gamma_{2} / 2 \pi \simeq 290 \mathrm{~Hz}$. The microwave circuit sustains two eigenmodes with frequencies $\omega_{1} / 2 \pi=5.63 \mathrm{GHz}$ and $\omega_{2} / 2 \pi=3.89 \mathrm{GHz}$ that couple roughly equally to both mechanical degrees of freedom [see Fig. 1(c)]. The two cavity modes couple to a line used for both read-out and excitation with external decay rates $\kappa_{1}^{e} / 2 \pi=406 \mathrm{kHz}$ and $\kappa_{2}^{e} / 2 \pi=115 \mathrm{kHz}$ (see Fig. 1) and internal decay rates $\kappa_{1}^{i} / 2 \pi=197 \mathrm{kHz}$ and $\kappa_{2}^{i} / 2 \pi=233 \mathrm{kHz}$, which makes cavity 1 overcoupled and cavity 2 undercoupled to the feedline. The total cavity linewidths $\kappa_{j}=\kappa_{j}^{i}+\kappa_{j}^{e}$ satisfy $\kappa_{j} / 2<\left|\Omega_{1}-\Omega_{2}\right|$ as supposed in the basic modeling, entailing that, up to a reasonable approximation, a given tone drives one process only. However, as the system is not very far into this regime, we calculate the effect of the offresonant coupling processes in Appendix $\mathrm{C}$ and find that they cause only a minor modification in our experimental situation.

The system is operated in a dilution refrigerator at a fixed temperature of $200 \mathrm{mK}$. The elevated temperature is used because we find that the mechanical frequencies fluctuate at the base temperature and an accurate drive-tone detuning cannot be maintained. Four synchronized independent generators, whose relative phase drift is less than $4^{\circ} / \mathrm{h}$, are used to pump the device.

We record the transfer parameters using a Rohde \& Schwartz ZVA-50 network analyzer that allows independent excitation and measurement frequencies. The probe is maintained at a very low power, $76 \mathrm{~dB}$ below the lowest pump power to ensure that probing does not modify the amplifier's behavior. An independently measured contribution from noise in the recorded frequency-converting response is subtracted to yield the pure transfer coefficients as explained in Appendix D. The pump and probe efficiencies around the cavity frequencies are determined by measuring a large frequency span around the cavities [see Fig. 2(a)] and are used to calibrate the transfer parameters of the amplifier following the method discussed in Appendix E. 

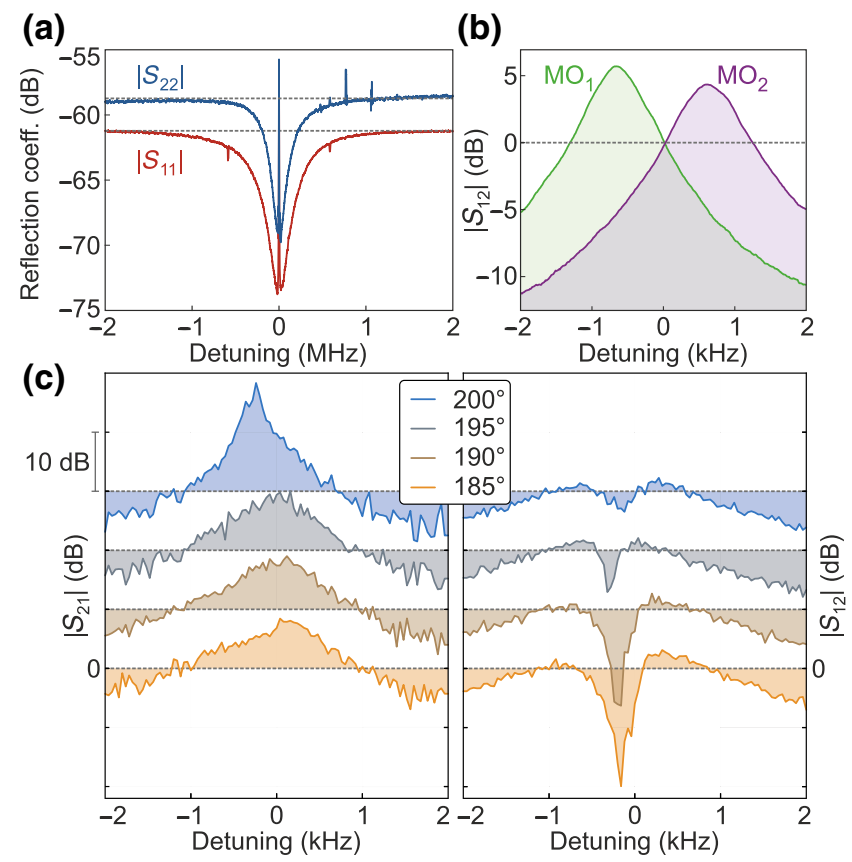

FIG. 2. Preparation of interference of amplification channels. (a) Uncalibrated reflection coefficient on cavity 1 (red) and cavity 2 (blue) over a broad frequency span around the resonance frequency of either cavity. Here two independent single-MO amplification processes are active (see the text). Cavity response is used to determine pump and read-out efficiencies through the whole cryogenic attenuation and amplification stage around each frequency range, indicated as dashed lines: $-61.2 \mathrm{~dB}$ around cavity $1,-58.8 \mathrm{~dB}$ around cavity 2 . Small features detuned by about $0.6 \mathrm{MHz}$ from cavity frequencies correspond to field oscillations at the mechanical frequency difference. Larger spurious peaks on $\left|S_{22}\right|$ above the cavity frequency arise from intermodulation of the pump tones in the measurement system. (b) Backward power transmission $\left|S_{12}\right|$ of single-MO amplifiers built from MO 1 only (green) and MO 2 only (purple), once balanced, with respect to frequency detuning from cavity 1 (the output frequency in this case). (c) Forward $\left|S_{21}\right|$ and backward $\left|S_{12}\right|$ transmission for different phase values $\Phi$ of one of the generators relative to the others. $\mathrm{MO}_{1}, \mathrm{MO} 1 ; \mathrm{MO}_{2}, \mathrm{MO}$.

\section{B. Directional amplifier}

To prepare the interference effect, we first drive each MO independently through its red and blue sidebands, reproducing two single-MO optomechanical reciprocal amplifiers as described in Ref. [40]. The blue-sideband drive powers are tuned to produce similar amplification for both single-MO amplifiers in the backward direction $S_{12}$, as shown in Fig. 2(b). In the experimental situation, the cooperativities used are different for each $\mathrm{MO}, C_{11}=1.27$ and $C_{12}=3.20$ for the red sidebands of $\mathrm{MO} 1$ and $\mathrm{MO}$ 2, respectively, and $C_{21}=1.33$ and $C_{22}=2.05$ for the blue sidebands. The pump tones are detuned by $\delta_{1} / 2 \pi=$ $-\delta_{2} / 2 \pi=600 \mathrm{~Hz}$ from the sidebands. We then turn on all pumps simultaneously, and the phase of one of them relative to the others is tuned to achieve destructive interference in the backward direction as shown in Fig. 2(c). Further fine-tuning of the frequencies, phase, and powers is generally required to compensate for slow phase and power drift of the generators and slight power dependence of the cavity frequencies.

The amplifier maps bijectively a frequency range around $\omega_{1} / 2 \pi$ to a frequency range centered on and mirrored about $\omega_{2} / 2 \pi$. A minimum backward transmission gain of $-21.3 \pm 1.1 \mathrm{~dB}$ is observed, as displayed in Fig. 3, while the forward gain reaches $9.4 \pm 1.1 \mathrm{~dB}$ at the same frequency. The uncertainty corresponds to the estimated maximum gain-calibration uncertainty (see Appendix E). The maximum nonreciprocity factor $\left|S_{21} / S_{12}\right|$ is therefore $30.7 \pm 2.2 \mathrm{~dB}$, which compares well with isolator implementations [36,37]. While perfect impedance matching is not enforced, the reflection attenuation $\left(S_{11}\right)$ reaches $3.9 \mathrm{~dB}$. The isolation bandwidth, defined as the frequency range where the backward transmitted power is attenuated by more than $3 \mathrm{~dB}$, is $500 \mathrm{~Hz}$, as expected since it is governed by the mechanical linewidths. If we take the amplification bandwidth as the frequency range amplified by more than half the maximum gain, the latter amounts to
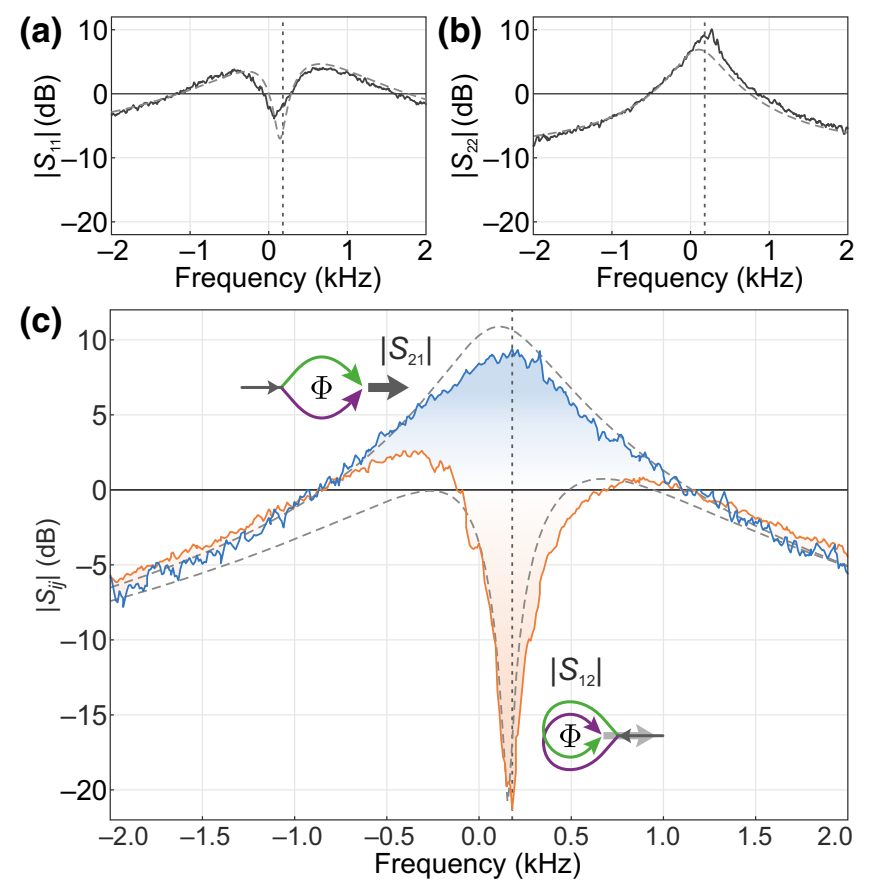

FIG. 3. $S$-parameter amplitudes of the amplifier with respect to frequency. (a) $\left|S_{11}\right|$, (b) $\left|S_{22}\right|$, and (c) $\left|S_{12}\right|$ (orange) and $\left|S_{21}\right|$ (blue). Dashed gray lines are simultaneous fits of all four data sets with the expressions in the text, where the phase is left free. The amplifier bijectively maps a frequency range around the resonance of one cavity to a mirrored range around the resonance of the other cavity: only one increasing frequency range around $\omega_{1} / 2 \pi$ is used as the horizontal axis of all plots for comparison. The maximum nonreciprocity frequency is indicated by a dashed vertical line. 


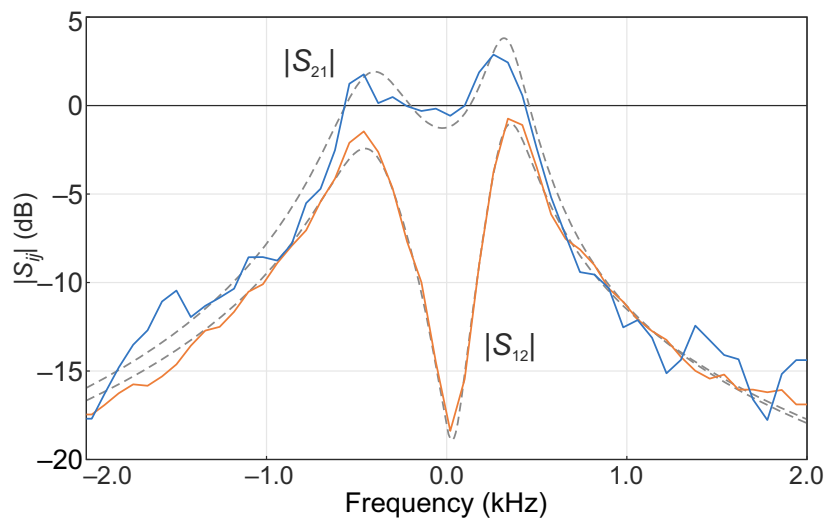

FIG. 4. $\left|S_{12}\right|$ (orange) and $\left|S_{21}\right|$ (blue) transfer parameters of a lossless isolator. Dashed gray lines are fits with the expressions in the text, where the phase is left free.

$675 \mathrm{~Hz}$, also on the order of magnitude of the mechanical linewidth because of the interference effect on the forward transfer.

\section{Lossless isolator}

In another configuration $\left(C_{11}=0.47, C_{12}=0.74\right.$, $C_{21}=0.84, \quad C_{22}=0.96, \quad \delta_{1} / 2 \pi=450 \mathrm{~Hz}, \quad \delta_{2} / 2 \pi=$ $-405 \mathrm{~Hz}$ ), the same device can be used as an isolator free from any insertion loss and whose forward and backward transfer gains are represented in Fig. 4. In this configuration, we obtain isolation by $18.0 \mathrm{~dB}$ of backwardpropagating signals.

\section{DISCUSSION}

Generation of interference between the paths linking the cavities requires mechanical oscillators with similar linewidths and optomechanical couplings. However, nonreciprocal multimode devices also require very unequal mechanical frequencies - in the sense that the spacing of the frequencies generally needs to be a rather large fraction of their value - to allow each mechanical oscillator to be addressed separately. This condition, which one could call "resolved sideband difference" (RSBD) analogously to "resolved sideband," appears to be very relevant as parasitic cross-driving of mechanical modes has been shown to significantly increase insertion loss of optomechanical isolators [37]. The present scheme balances these opposite requirements of equal couplings but different frequencies by involving two separate drum resonators, contrary to what was done previously in Refs. [36-38], where different eigenmodes of the same resonator were used. For this reason this scheme is also immune to any direct coupling between modes through geometrical nonlinearities.

While the current device represents a technological step forward in multimode optomechanical applications, the
RSBD condition was attained here at the price of our deliberately reducing the external decay rates of the cavities, thus departing from the ideal far-overcoupled situation. The question of cavity dissipation might become essential in the description of real multimode optomechanical systems, which is why in this article we attempt to draw particular attention to them. On the experimental side, achieving the RSBD condition while maintaining overcoupled cavities is one of the next endeavors to manufacture high-quality nonreciprocal devices from multimode optomechanical systems. Another goal is to carefully characterize the noise properties of the amplifier. One can realistically achieve an added noise near the quantum limit; however, this requires operation at temperatures appreciably lower than the temperature we used in the current experiment for stability reasons.

\section{CONCLUSIONS}

We report on a multimode optomechanical directional amplifier, demonstrating both high isolation between forward and backward transfer and gain in the forward direction. We also demonstrate a nonreciprocal optomechanical device using two separate mechanical resonators. Moreover, we formulate some guidelines for the design of such devices in the real situation where dissipation rates of cavities compare with other system frequencies.

\section{ACKNOWLEDGMENTS}

This work was supported by the Academy of Finland (Grants No. 250280, No. 308290, No. 307757, and No. 312296), by the European Research Council (CAVITYQPD, Grant Agreement No. 615755), by the Aalto Centre for Quantum Engineering, and by the EU Horizon 2020 research and innovation program under Grant Agreement No. 732894 (FET Proactive HOT). We acknowledge the facilities and technical support of the Otaniemi research infrastructure for Micro- and Nanotechnologies (OtaNano).

\section{APPENDIX A: THEORY}

\section{General expression for the coupling matrices}

Creation and annihilation operators for cavity $j$ are denoted $a_{j}$ and $a_{j}^{\dagger}$, and the same operators for $\mathrm{MO} j$ are denoted $b_{j}$ and $b_{j}^{\dagger}$. The total Hamiltonian of the system is given in Eq. (1).

Following Ref. [43], we consider for generality a morecomplete pumping scheme than was used in the article. In the complete scheme, each cavity $i$ can be driven by four tones with frequencies $\left[\omega_{i} \pm\left(\Omega_{j}+\delta_{j}\right)\right]_{j=1,2}$; that is, the frequencies of the two sidebands of each of the two oscillators. The photon field $a_{i}(t)$ in cavity $i$ can be decomposed into a coherent driven part of amplitude $\alpha_{i}(t)$ oscillating at 
pump frequencies and a fluctuation $\delta a_{i}(t)$ :

$$
\begin{aligned}
& a_{i}(t)=e^{-i \omega_{i} t} \alpha_{i}(t)+\delta a_{i}(t), \\
& \alpha_{i}(t) \equiv \sum_{j} \alpha_{i j-} e^{+i\left(\Omega_{j}+\delta_{j}\right) t}+\alpha_{i j+} e^{-i\left(\Omega_{j}+\delta_{j}\right) t},
\end{aligned}
$$

where we introduced the intracavity field amplitudes at the frequencies of the red-detuned (blue-detuned) sidebands $\alpha_{i j-}\left(\alpha_{i j+}\right)$. The Hamiltonian can be linearized in the fluctuations $\delta a_{i}$ in a standard semiclassical approach. The quantum photon-field fluctuation $\delta a_{i}$ is now renamed $a_{i}$ for convenience. In the frame rotating with

$$
H_{0} / \hbar=\sum_{i} \omega_{i} a_{i}^{\dagger} a_{i}+\sum_{j}\left(\Omega_{j}+\delta_{j}\right) b_{j}^{\dagger} b_{j},
$$

the linearized Hamiltonian is rewritten

$$
\begin{aligned}
H_{\mathrm{rot}} / \hbar= & -\sum_{j} \delta_{j} b_{j}^{\dagger} b_{j} \\
& -\sum_{i j} g_{i j}\left[\alpha_{i}^{*}(t) a_{i}\left(b_{j} e^{-i\left(\Omega_{j}+\delta_{j}\right) t}+b_{j}^{\dagger} e^{+i\left(\Omega_{j}+\delta_{j}\right) t}\right)+\text { H.c. }\right] .
\end{aligned}
$$

The hierarchy of the system frequencies is $\delta_{1}, \delta_{2} \simeq$ $\gamma_{1}, \gamma_{2} \ll \kappa_{1}, \kappa_{2}<\left|\Omega_{2}-\Omega_{1}\right|<\Omega_{1}, \Omega_{2} \ll \omega_{1}, \omega_{2}$. The system is in the resolved-sideband regime. However, some terms in the last equation are also oscillating at the mechanical frequency difference, which is greater than the linewidths of the cavities by only some tens of percent.

Ignoring any term oscillating faster than $\gamma_{j}$ in the evolution equation for phononic operators $b_{j}$ and any term oscillating faster than $\kappa_{i}$ in the evolution equation for photonic operators $a_{i}$, one obtains the following effective time-independent Hamiltonian:

$$
\begin{aligned}
H_{\mathrm{rot}} / \hbar= & -\sum_{j} \delta_{j} b_{j}^{\dagger} b_{j}-\sum_{i j} g_{i j}\left(\alpha_{i j+} a_{i}^{\dagger} b_{j}^{\dagger}+\alpha_{i j-} a_{i}^{\dagger} b_{j}\right. \\
& \left.+\alpha_{i j+}^{*} a_{i} b_{j}+\alpha_{i j-}^{*} a_{i} b_{j}^{\dagger}\right) .
\end{aligned}
$$

The Fourier transform of the evolution equations gives

$$
\begin{aligned}
b_{j}(\omega)= & \chi_{m, j}(\omega)\left\{i \sum_{i=1}^{2} g_{i j}\left[\alpha_{i j-}^{*} a_{i}(\omega)+\alpha_{i j+} a_{i}^{\dagger}(\omega)\right]\right. \\
& \left.+\sqrt{\gamma_{j}} b_{\mathrm{in}, j}(\omega)\right\}, \\
a_{i}(\omega)= & \chi_{c, i}(\omega)\left\{i \sum_{j=1}^{2} g_{i j}\left[\alpha_{i j} b_{j}^{\dagger}(\omega)+\alpha_{i j-} b_{j}(\omega)\right]\right. \\
& \left.+\sqrt{\kappa_{i}^{i}} a_{\mathrm{in}, i}^{i}(\omega)+\sqrt{\kappa_{i}^{e}} a_{\mathrm{in}, i}^{e}(\omega)\right\},
\end{aligned}
$$

with the effective mechanical and cavity susceptibilities

$$
\begin{aligned}
\chi_{m, j}(\omega) & \equiv\left[\frac{\gamma_{j}}{2}-i\left(\omega+\delta_{j}\right)\right]^{-1} \\
\chi_{c, i}(\omega) & \equiv\left(\frac{\kappa_{i}}{2}-i \omega\right)^{-1} .
\end{aligned}
$$

Here we used the same convention for operators and functions: for any annihilation operator $[c(\omega)]^{\dagger}=c^{\dagger}(-\omega)$ and for any function $[f(\omega)]^{*}=f^{*}(-\omega)$.

The effective couplings to each cavity that appear in the previous equations are now written $G_{i j} \equiv g_{i j} \alpha_{i j-}$ and $J_{i j} \equiv g_{i j} \alpha_{i j+} . G_{i j}\left(J_{i j}\right)$ therefore corresponds to the enhanced optomechanical coupling from red-sideband (blue-sideband) pumping; the first index is attached to the cavity and the second one is attached to the MO.

We now simplify the problem involving four photonic and four phononic operators to a problem involving only one photonic vector operator and one phononic vector operator. To this end we define $A(\omega) \equiv$ $\left(a_{1}(\omega) \quad a_{1}^{\dagger}(\omega) \quad a_{2}(\omega) \quad a_{2}^{\dagger}(\omega)\right)^{T} \quad$ and $\quad B \equiv\left(b_{1}(\omega)\right.$ $\left.b_{1}^{\dagger}(\omega) b_{2}(\omega) b_{2}^{\dagger}(\omega)\right)^{T}$ and the corresponding $A_{\text {in }}^{e}(\omega)$, $A_{\text {in }}^{i}(\omega)$, and $B_{\text {in }}(\omega)$. We also define the susceptibility matrices for the cavities and mechanical oscillators,

$$
\begin{aligned}
\Xi_{c}(\omega) & \equiv \operatorname{diag}\left[\chi_{c, 1}(\omega), \chi_{c, 1}^{*}(\omega), \chi_{c, 2}(\omega), \chi_{c, 2}^{*}(\omega)\right], \\
\Xi_{m}(\omega) & \equiv \operatorname{diag}\left[\chi_{m, 1}(\omega), \chi_{m, 1}^{*}(\omega), \chi_{m, 2}(\omega), \chi_{m, 2}^{*}(\omega)\right],
\end{aligned}
$$

the total cavity and mechanical decay-rate vectors,

$$
K \equiv \operatorname{diag}\left(\kappa_{1}, \kappa_{1}, \kappa_{2}, \kappa_{2}\right), \quad \Gamma \equiv \operatorname{diag}\left(\gamma_{1}, \gamma_{1}, \gamma_{2}, \gamma_{2}\right),
$$

and $K^{i}$ and $K^{e}$, the internal cavity and external cavity decay-rate matrices, with analogous definitions. Having established this matrix formalism, we can now rewrite the two coupled equations for photonic and phononic fields:

$$
\begin{aligned}
& A(\omega)=\Xi_{c}(\omega)\left[\mathcal{G} B(\omega)+\sqrt{K^{e}} A_{\text {in }}^{e}(\omega)+\sqrt{K^{i}} A_{\text {in }}^{i}(\omega)\right], \\
& B(\omega)=\Xi_{m}(\omega)\left[\mathcal{H} A(\omega)+\sqrt{\Gamma} B_{\text {in }}(\omega)\right],
\end{aligned}
$$

where the coupling matrices are

$$
\begin{gathered}
\mathcal{G} \equiv i\left(\begin{array}{cccc}
G_{11} & J_{11} & G_{12} & J_{12} \\
-J_{11}^{*} & -G_{11}^{*} & -J_{12}^{*} & -G_{12}^{*} \\
G_{21} & J_{21} & G_{22} & J_{22} \\
-J_{21}^{*} & -G_{21}^{*} & -J_{22}^{*} & -G_{22}^{*}
\end{array}\right), \\
\mathcal{H} \equiv i\left(\begin{array}{cccc}
G_{11}^{*} & J_{11} & G_{21}^{*} & J_{21} \\
-J_{11}^{*} & -G_{11} & -J_{21}^{*} & -G_{21} \\
G_{12}^{*} & J_{12} & G_{22}^{*} & J_{22} \\
-J_{12}^{*} & -G_{12} & -J_{22}^{*} & -G_{22}
\end{array}\right) .
\end{gathered}
$$


Replacing the phononic matrix operator in the photonic matrix equation, one gets the expression for photonic fields perturbed by the coupling to phononic fields:

$$
\begin{aligned}
& {\left[\Xi_{c}(\omega)^{-1}-\mathcal{G} \Xi_{m}(\omega) \mathcal{H}\right] A(\omega)} \\
& \quad=\sqrt{K^{e}} A_{\text {in }}^{e}(\omega)+\sqrt{K^{i}} A_{\text {in }}^{i}(\omega)+\mathcal{G} \Xi_{m}(\omega) \sqrt{\Gamma} B_{\text {in }}(\omega) .
\end{aligned}
$$

Here we get an explicit expression for the coupling matrix as defined in the main text,

$$
T(\omega) \equiv-\mathcal{G} \Xi_{m}(\omega) \mathcal{H},
$$

which contains coupling amplitudes for all phononmediated photon-photon couplings. Furthermore, we identify as in the main text the global photonic system susceptibility:

$$
\begin{aligned}
\chi(\omega) & \equiv \Xi_{c}(\omega)^{-1}-\mathcal{G} \Xi_{m}(\omega) \mathcal{H} \\
& =\Xi_{c}(\omega)^{-1}+T(\omega) .
\end{aligned}
$$

We also get the expression for the matrix $U(\omega)$ in the main text that characterizes cavity heating due to mechanical thermal or quantum noise:

$$
U(\omega) \equiv \mathcal{G} \Xi_{m}(\omega) \sqrt{\Gamma}
$$

\section{Phase-insensitive directional amplifier}

In the experimental case, cavity 1 is pumped only on red sidebands so that $J_{1 i}=0$ and cavity 2 is pump only on blue sidebands so that $G_{2 i}=0$, which yields the hollow $T(\omega)$ coupling matrix given in the main text, with coefficients

$$
\begin{aligned}
& T_{11}(\omega)=\left|G_{11}\right|^{2} \chi_{m, 1}(\omega)+\left|G_{12}\right|^{2} \chi_{m, 2}(\omega), \\
& T_{12}(\omega)=G_{11} J_{21} \chi_{m, 1}(\omega)+G_{12} J_{22} \chi_{m, 2}(\omega), \\
& T_{21}(\omega)=-\left[G_{11}^{*} J_{21}^{*} \chi_{m, 1}(\omega)+G_{12}^{*} J_{22}^{*} \chi_{m, 2}(\omega)\right], \\
& T_{22}(\omega)=-\left[\left|J_{21}\right|^{2} \chi_{m, 1}(\omega)+\left|J_{22}\right|^{2} \chi_{m, 2}(\omega)\right],
\end{aligned}
$$

which leads to the expressions given in the main text in terms of cooperativities, decay rates, and pump relative phases.

\section{APPENDIX B: AMPLIFIER PARAMETERS}

\section{1. $S_{\text {opt }}$ elements}

$S_{\text {opt }}$ has the same structure as the $T$ matrix; that is,

$$
S_{\mathrm{opt}}(\omega)=\left(\begin{array}{cccc}
S_{11}(\omega) & 0 & 0 & S_{12}(\omega) \\
0 & S_{11}^{*}(\omega) & S_{12}^{*}(\omega) & 0 \\
0 & S_{21}^{*}(\omega) & S_{22}^{*}(\omega) & 0 \\
S_{21}(\omega) & 0 & 0 & S_{22}(\omega)
\end{array}\right)
$$

with the following coefficients:

$$
\begin{aligned}
& S_{11}=1-\kappa_{1}^{e} \chi_{c, 1} \frac{1+\chi_{c, 2} T_{22}}{D}, \\
& S_{12}=\sqrt{\kappa_{1}^{e} \kappa_{2}^{e}} \frac{\chi_{c, 1} \chi_{c, 2} T_{12}}{D}, \\
& S_{21}=\sqrt{\kappa_{1}^{e} \kappa_{2}^{e}} \frac{\chi_{c, 1} \chi_{c, 2} T_{21}}{D}, \\
& S_{22}=1-\kappa_{2}^{e} \chi_{c, 2} \frac{1+\chi_{c, 1} T_{11}}{D},
\end{aligned}
$$

with the common denominator

$$
D=\left(1+\chi_{c, 1} T_{11}\right)\left(1+\chi_{c, 2} T_{22}\right)-\chi_{c, 1} \chi_{c, 2} T_{12} T_{21} .
$$

\section{Impedance matching}

The reflection coefficient is

$$
\left|S_{11}(0)\right|=1-\frac{2 r_{1}}{2 C_{1} /\left(1+4 \delta^{2}\right)+1} .
$$

There is no reflection on the input cavity if this coefficient is 0 ; that is, for $r_{1} \neq \frac{1}{2}$ (input cavity not critically coupled, $\left.\kappa_{1}^{e} \neq \kappa_{1}^{i}\right)$, and

$$
\delta=\frac{1}{2} \sqrt{\frac{2 C_{1}}{1-2 r_{1}}-1}=\frac{\sqrt{2 C_{1}\left[\kappa_{1} /\left(\kappa_{1}^{e}-\kappa_{1}^{i}\right)\right]-1}}{2} .
$$

For nonlossy cavities, one recovers the criterion from the proposal $\delta=\left(2 C_{1}-1\right) / 2$, which is possible for $C_{1}>0.5$. However, this criterion can never be met if the input cavity is undercoupled $\kappa_{1}^{i} \leq \kappa_{1}^{e}$, and is met only for

$$
C_{1}>0.5 \frac{\kappa_{1}^{e}-\kappa_{1}^{i}}{\kappa_{1}}
$$

in the general lossy case. This indicates that, unsurprisingly, as losses will require increased red-sideband cooperativities, they will also require increased blue-sideband cooperativities for some gain to be observed, so gain is more difficult to obtain with lossy cavities if the impedance-matching condition is to be met.

\section{Isolation}

The isolation condition $S_{12}(0)=0$ is achieved when $T_{12}(0)=0$; that is,

$$
e^{i\left(\theta_{11}+\theta_{21}-\theta_{12}-\theta_{22}\right)} \frac{\gamma_{1}}{\gamma_{1} / 2-i \delta_{1}}=-\frac{\gamma_{2}}{\gamma_{2} / 2-i \delta_{2}} .
$$

Hence there are two conditions concerning the modulus and phase of the members of this equality. The condition 
on the modulus reduces to

$$
\frac{1}{4}+\frac{\delta_{1}^{2}}{\gamma_{1}^{2}}=\frac{1}{4}+\frac{\delta_{2}^{2}}{\gamma_{2}^{2}} \rightarrow \delta_{1}^{2} \gamma_{2}^{2}=\delta_{2}^{2} \gamma_{1}^{2}
$$

With the proposition [43] $\delta_{1}=\delta \gamma_{1}$ and $\delta_{2}=-\delta \gamma_{2}$ and by denoting $\Phi=\theta_{11}+\theta_{21}-\theta_{12}-\theta_{22}$, the previous phase equality is equivalent to

$$
\Phi=\arg \left(\frac{-1+2 i \delta}{1+2 i \delta}\right) .
$$

This condition can be met regardless of cavity losses and even if the isolation conditions on $\delta$ and $\Phi$ are independent of the losses.

\section{Gain}

The power gain of the amplifier is

$$
\begin{aligned}
\left|S_{21}(0)\right|^{2} & =\left|\frac{T_{21} \sqrt{\kappa_{1}^{e} \kappa_{2}^{e}} \chi_{c 1} \chi_{c 2}}{1+T_{11} \chi_{c 1}+T_{22} \chi_{c 2}+T_{11} T_{22} \chi_{c 1} \chi_{c 2}}\right|^{2} \\
& =r_{1} r_{2} \frac{64 \delta^{2} C_{1} C_{2}\left(1+4 \delta^{2}\right)^{2}}{\left(2 C_{1}+1+4 \delta^{2}\right)^{2}\left(1+4 \delta^{2}-2 C_{2}\right)^{2}},
\end{aligned}
$$

where we defined $r_{j} \equiv \kappa_{j}^{e} /\left(\kappa_{j}^{e}+\kappa_{j}^{i}\right)$. The impedancematching condition in the case of a lossy input cavity is $1+$ $4 \delta^{2}=2 C_{1} /\left(1-2 r_{1}\right)$. The gain in the impedance-matched condition is then

$$
\left|S_{21}(0)\right|^{2}=r_{1} r_{2}\left(\frac{2 r_{1}-1}{r_{1}}\right)^{2} \frac{2 C_{2}\left(2 C_{1}+1-2 r_{1}\right)}{\left[C_{1}+C_{2}\left(1-2 r_{1}\right)\right]^{2}} .
$$

In the case $C_{1}=C_{2}$ and the limit $C_{1}, C_{2} \rightarrow \infty$, the gain is

$$
\lim _{C_{1}, C_{2} \rightarrow \infty}\left|S_{21}(0)\right|^{2}=\frac{2 r_{2}}{r_{1}} \frac{\left(2 r_{1}-1\right)^{2}}{1-r_{1}} .
$$

For $r_{1}>0.5$ (required by the impedance-matching condition) and $r_{2}>0$, this is a growing function of both $r_{1}$ and $r_{2}$ (plotted in Fig. 5). From Fig. 5 it becomes clear that the internal losses of the input cavity are much more deleterious to the amplifier gain than those of the output cavity.

\section{Added noise}

The output field is written

$$
A_{\mathrm{out}}=M_{c}^{e} A_{\mathrm{in}}^{e}+M_{c}^{i} A_{\mathrm{in}}^{i}+M_{m} B_{\mathrm{in}}^{i},
$$

where we defined three matrices of interest, $M_{c}^{e} \equiv S_{\text {opt }}$, $M_{c}^{i} \equiv-\sqrt{K^{e}} \chi \sqrt{K^{i}}$, and $M_{m} \equiv-\sqrt{K^{e}} \chi U$, that characterize how noise due to the thermal occupations of the cavity

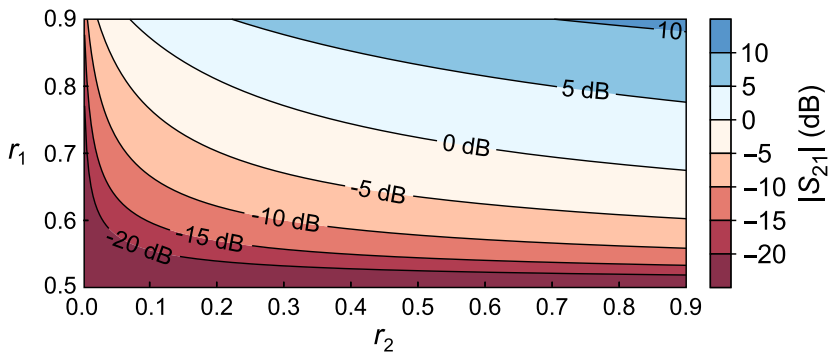

FIG. 5. Gain $\left|S_{21}(0)\right|^{2}$ in the limit of high cooperativities, $C_{1}=C_{2}$ and in the impedance-matched case as a function of $r_{1}=\kappa_{1}^{e} / \kappa_{1}$ and $r_{2}=\kappa_{2}^{e} / \kappa_{2}$.

modes and the mechanical oscillators is distributed on the two ports of the device.

The total output spectrum displays two frequency ranges of interest, around each cavity's resonance, which we can identify as the output spectrum at each port of the device. We can then define the total output spectrum at port 2 (the output port) as

$$
S_{\mathrm{out}, 2}(\omega) \equiv \frac{1}{2}\left\langle a_{\mathrm{out}, 2}^{\dagger}(\omega) a_{\mathrm{out}, 2}(-\omega)+a_{\mathrm{out}, 2}(\omega) a_{\mathrm{out}, 2}^{\dagger}(-\omega)\right\rangle,
$$

and similarly for port 1 .

The added noise is due to quantum noise, as well as excess thermal occupation in both the cavities and the MOs. The cavity noise can have two origins; namely, internal and external noise, represented by source terms $A_{\text {in }}^{i}(\omega)$ and $A_{\text {in }}^{e}(\omega)$ with corresponding thermal occupations $n_{c, 1}^{i}, n_{c, 2}^{i}$ and $n_{c, 1}^{e}, n_{c, 2}^{e}$, summing up to the total cavity occupations $n_{c 1}$ and $n_{c 2}$.

Eventually, the external thermal noise can be safely ignored in a typical experimental case near the quantum regime. The internal noise, however, can reach thermal occupations on the order of one quantum in a typical experiment due to technical heating, and it needs to be considered. We make the standard assumption that all noises driving the system are uncorrelated, so the final contributions are only due to terms such as $\left\langle a_{\mathrm{in}, j}^{i \dagger} a_{\mathrm{in}, j}^{i}\right\rangle \equiv n_{c, j}^{i}$, $\left\langle a_{\mathrm{in}, j}^{e \dagger} a_{\mathrm{in}, j}^{e}\right\rangle \equiv n_{c, j}^{e}$, and $\left\langle b_{\mathrm{in}, k}^{\dagger} b_{\mathrm{in}, k}\right\rangle \equiv n_{m, k}$, where $n_{m, k}$ is $\mathrm{MO}$ k's thermal occupancy. We can then separate contributions to the output noise arising from the cavities' internal and external noise and from mechanical noise: $S_{\text {out }, 2}=$ $S_{\text {out }, 2}^{i}+S_{\text {out }}^{e}+S_{\text {out }}^{m}$ (and similar definitions for port 1).

The matrix $M_{c}^{i}$ has the same structure and symmetry as $S_{\text {opt }}$ and $T$. Its elements, denoted in the same way as those of $S_{\text {opt }}$ and $T$, are

$$
\begin{aligned}
& M_{c 11}^{i}=\sqrt{\kappa_{1}^{e} \kappa_{1}^{i}} \chi_{c, 1} \frac{1+\chi_{c, 2} T_{22}}{D}, \\
& M_{c 12}^{i}=\sqrt{\kappa_{1}^{e} \kappa_{2}^{i}} \frac{\chi_{c, 1} \chi_{c, 2} T_{12}}{D},
\end{aligned}
$$




$$
\begin{aligned}
& M_{c 21}^{i}=\sqrt{\kappa_{1}^{i} \kappa_{2}^{e}} \frac{\chi_{c, 1} \chi_{c, 2} T_{21}}{D}, \\
& M_{c 22}^{i}=\sqrt{\kappa_{2}^{e} \kappa_{2}^{i}} \chi_{c, 2} \frac{1+\chi_{c, 1} T_{11}}{D},
\end{aligned}
$$

with the same common denominator as $S_{\text {opt }}$ elements [Eq. (B3)]. On resonance, the noise at the input and output ports from the cavity modes' thermal occupation is then

$$
\begin{aligned}
& S_{\text {out }, 1}^{i}=\left|M_{c 11}^{i}\right|^{2}\left(n_{c, 1}^{i}+\frac{1}{2}\right)+\left|M_{c 12}^{i}\right|^{2}\left(n_{c, 2}^{i}+\frac{1}{2}\right), \\
& S_{\text {out }, 2}^{i}=\left|M_{c 21}^{i}\right|^{2}\left(n_{c, 1}^{i}+\frac{1}{2}\right)+\left|M_{c 22}^{i}\right|^{2}\left(n_{c, 2}^{i}+\frac{1}{2}\right),
\end{aligned}
$$

where $n_{c, 1}^{i}\left(n_{c, 2}^{i}\right)$ is the thermal occupation of cavity 1 (cavity 2). Noise from the external type of heating follows the same rule, with superscript $e$ instead of $i$. For $M_{c 12}^{i}=0$ (and also $M_{c 12}^{e}=S_{12}=0$ ) at resonance the same destructive interference that suppresses backward-propagating signal suppresses all backward-propagating cavity noise, regardless of the quality of the cavities. The other elements of $M_{c}^{i}$ on resonance are

$$
\begin{aligned}
& M_{c 11}^{i}=\frac{2 \sqrt{r_{1}\left(1-r_{1}\right)}\left(1+4 \delta^{2}\right)}{2 C_{1}+4 \delta^{2}+1}, \\
& M_{c 21}^{i}=S_{21}(0) \sqrt{\frac{1-r_{1}}{r_{1}}}, \\
& M_{c 22}^{i}=-\frac{2 \sqrt{r_{2}\left(1-r_{2}\right)}\left(1+4 \delta^{2}\right)}{-2 C_{2}+4 \delta^{2}+1} .
\end{aligned}
$$

Finally, in the case of impedance matching,

$$
\begin{aligned}
M_{c 11}^{i} & =\sqrt{\frac{1-r_{1}}{r_{1}}}, \\
M_{c 21}^{i} & =S_{21}(0) \sqrt{\frac{1-r_{1}}{r_{1}}}, \\
M_{c 22}^{i} & =-\frac{2 C_{1} \sqrt{r_{2}\left(1-r_{2}\right)}}{C_{1}+\left(2 r_{1}-1\right) C_{2}} .
\end{aligned}
$$

We now turn to the calculation of the $M_{m}$ matrix. It includes the matrix $U \equiv \mathcal{G} \Xi_{m} \sqrt{\Gamma}$ [see Eq. (A15)]. Matrices $\sqrt{\Gamma}$ and $\Xi_{m}$ are diagonal matrices, so $U$ conserves the structure and symmetry of $\mathcal{G}$ :

$$
U=\left(\begin{array}{cccc}
U_{11} & 0 & U_{12} & 0 \\
0 & U_{11}^{*} & 0 & U_{12}^{*} \\
0 & U_{21}^{*} & 0 & U_{22}^{*} \\
U_{21} & 0 & U_{22} & 0
\end{array}\right) .
$$

This matrix has relatively simple elements in the case where isolation is implemented:

$$
\begin{aligned}
& U_{11}=-U_{12}^{*}=-\sqrt{\frac{C_{1} \kappa_{1}}{1+4 \delta^{2}}}, \\
& U_{21}=-U_{22}^{*}=\frac{\sqrt{C_{2} \kappa_{2}}}{2 \delta+i} .
\end{aligned}
$$

The structure of $U$ from Eq. (B19) is transferred to $M_{m}$, whose elements are then denoted following the same pattern and are

$$
\begin{aligned}
& M_{m 11}=\sqrt{\kappa_{1}^{e}} \chi_{c, 1} \frac{\left(1+\chi_{c, 2} T_{22}\right) U_{11}-\chi_{c, 2} T_{12} U_{21}}{D}, \\
& M_{m 12}=\sqrt{\kappa_{1}^{e}} \chi_{c, 1} \frac{\left(1+\chi_{c, 2} T_{22}\right) U_{12}-\chi_{c, 2} T_{12} U_{22}}{D}, \\
& M_{m 21}=\sqrt{\kappa_{2}^{e}} \chi_{c, 2} \frac{\left(1+\chi_{c, 1} T_{11}\right) U_{21}-\chi_{c, 1} T_{21} U_{11}}{D}, \\
& M_{m 22}=\sqrt{\kappa_{2}^{e}} \chi_{c, 2} \frac{\left(1+\chi_{c, 1} T_{11}\right) U_{22}-\chi_{c, 1} T_{21} U_{12}}{D},
\end{aligned}
$$

with again the same denominator $D$ [Eq. (B3)]. With this notation, the contribution of mechanical noise to the noise at input and output ports is

$$
\begin{aligned}
& S_{\text {out }, 1}^{m}=\left|M_{m 11}\right|^{2}\left(n_{m, 1}+\frac{1}{2}\right)+\left|M_{m 12}\right|^{2}\left(n_{m, 2}+\frac{1}{2}\right), \\
& S_{\text {out }, 2}^{m}=\left|M_{m 21}\right|^{2}\left(n_{m, 1}+\frac{1}{2}\right)+\left|M_{m 22}\right|^{2}\left(n_{m, 2}+\frac{1}{2}\right),
\end{aligned}
$$

where $n_{m, 1}\left(n_{m, 2}\right)$ is the thermal occupation of mechanical mode 1 (mechanical mode 2). The coefficients in Eq. (B22) are

$$
\begin{aligned}
M_{m 11} & =\frac{2 \sqrt{C_{1}} \sqrt{r_{1}\left(1+4 \delta^{2}\right)}}{\left(2 C_{1}+1+4 \delta^{2}\right)}=-M_{m 12}^{*}, \\
M_{m 21} & =\frac{2 \sqrt{C_{2} r_{2}}(2 \delta+i)\left(1+2 C_{1}+4 i \delta-4 \delta^{2}\right)}{\left(1+2 C_{1}+4 \delta^{2}\right)\left(1-2 C_{2}+4 \delta^{2}\right)} \\
& =-M_{m 22}^{*} .
\end{aligned}
$$

In the impedance-matched case they become

$$
\begin{aligned}
M_{m 11} & =-M_{m 12}=-\sqrt{\frac{2 r_{1}-1}{2 r_{1}}}, \\
M_{m 21} & =-\frac{\left(2 r_{1}-1\right) \sqrt{C_{2} r_{2}}\left[\sqrt{2 C_{1} /\left(2 r_{1}-1\right)-1}\left(1 / r_{1}-1\right)-i\right]}{C_{1}-C_{2}\left(2 r_{1}-1\right)} \\
& =-M_{m 22}^{*} .
\end{aligned}
$$

The noise added by the amplifier is defined as the total noise at the output port, scaled in terms of the number of 
photons at the input port, minus the external noise injected at the input port:

$$
S_{\mathrm{add}} \equiv \frac{S_{\mathrm{out}, 2}^{e}+S_{\mathrm{out}, 2}^{i}+S_{\mathrm{out}, 2}^{m}}{\left|S_{21}\right|^{2}}-S_{\mathrm{in}, 1}^{e}
$$

To show the impact of cavity losses on added noise, Fig. 6 presents the contribution of each mode occupancy to the added noise in the situation $C_{1}=C_{2}$ and where impedance matching is enforced. As noticed in the previous section, the gain $\left|S_{21}\right|^{2}$ collapses for critical input-cavity coupling $r_{1} \rightarrow 0.5$ : any noise from the output cavity is then interpreted as a large number of photons from the input cavity, which reinforces the argument to use low-loss input cavities. Meanwhile, $r_{2}$ has a very little impact. However, in the case of lossy cavities, the main source of noise is typically the output-cavity occupancy $\left(M_{c 22}^{i}>\left\{M_{c 21}^{i}, M_{m 21}\right\}\right)$, which increases with $\kappa_{2}^{i}$ : a low-loss and therefore little-thermallyexcited output cavity is still desirable even though the reason for this is less directly observable with this analysis.

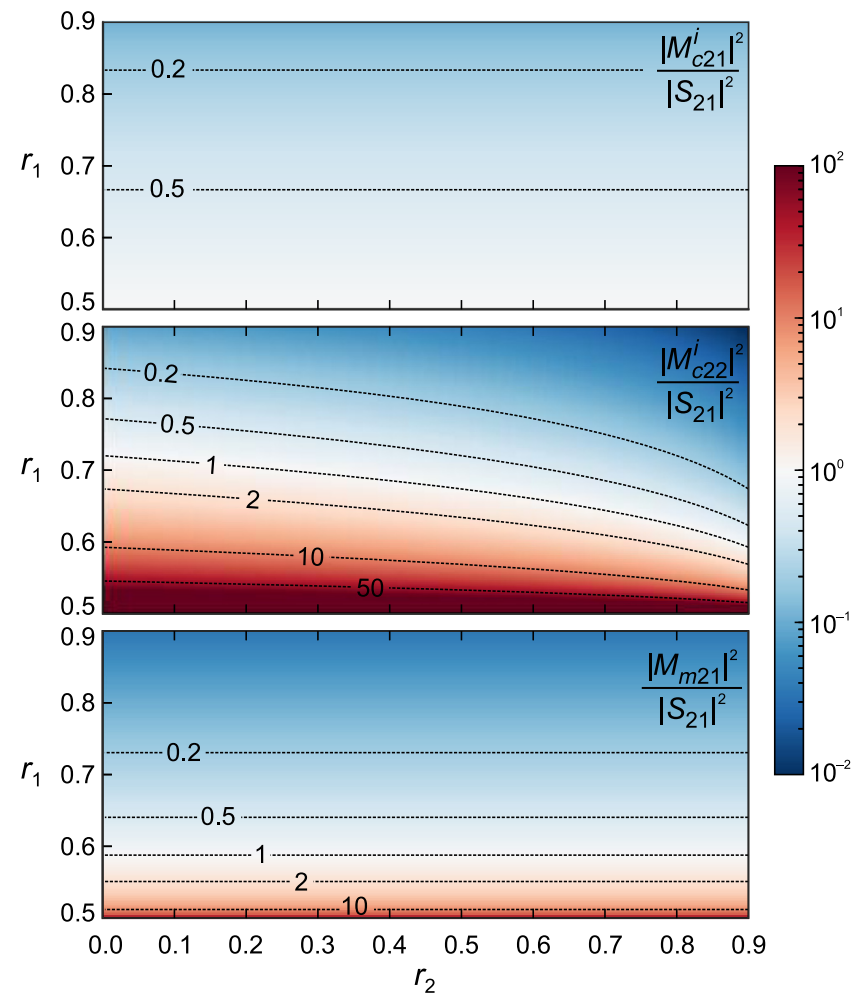

FIG. 6. Contributions to added noise by cavity and mechanical noise: coefficients of the occupancies in the added noise of the input cavity (top), of the output cavity (middle), and of either mechanical mode (bottom) in the case of an impedance-matched device, lossy cavities, $C_{1}=C_{2}$, and large cooperativities where these coefficients do not depend on cooperativities anymore.
Finally we comment on the lossless case $r_{1}=r_{2}=1$ as well as the impedance-matched case. One obtains

$$
M_{c 11}^{i}=M_{c 12}^{i}=M_{c 21}^{i}=M_{c 22}^{i}=0
$$

which is of course expected since there is no coupling channel to the environment if $\kappa^{i}=0$.

The impact of cavity heating from the external line is

$$
\left|M_{c 11}^{e}\right|^{2}=\left|M_{c 12}^{e}\right|^{2}=0
$$

that is, no cavity noise propagates back to port 1 ,

$$
\left|M_{c 21}^{e}\right|^{2}=\left|S_{21}\right|^{2}
$$

so cavity-1 noise is transmitted and amplified at port 2 with the same gain as the signal (i.e., it does not contribute to added noise), and

$$
\left|M_{c 22}^{e}\right|^{2}=\left|S_{22}\right|^{2}=\frac{\left(C_{1}+C_{2}\right)^{2}}{\left(C_{1}-C_{2}\right)^{2}}
$$

so external heating of cavity 2 does contribute to the added noise. The impact of mechanical noise is

$$
\left|M_{m 11}\right|^{2}=\left|M_{m 12}\right|^{2}=\frac{1}{2}
$$

at port 1 , and

$$
\left|M_{m 21}\right|^{2}=\left|M_{m 22}\right|^{2}=\frac{C_{2}}{\left(C_{1}-C_{2}\right)^{2}},
$$

which contributes to the noise added at port 2. Scaling this noise by the forward gain $S_{21}$, one recovers the expressions given in the theoretical proposal [43] for the added noise in the case of lossless cavities,

$$
S_{\mathrm{add}}=\frac{1}{4 C_{1}}\left(n_{m, 1}+n_{m, 2}+1\right)+\frac{\left(C_{1}+C_{2}\right)^{2}}{4 C_{1} C_{2}}\left(n_{c, 2}+\frac{1}{2}\right),
$$

and for the noise at port 1 ,

$$
S_{\mathrm{back}} \equiv S_{\mathrm{out}, 1}=\frac{1}{2}\left(n_{m, 1}+n_{m, 2}+1\right)
$$

\section{APPENDIX C: PARASITIC DRIVING OF MECHANICAL OSCILLATORS}

In the case where the RSBD condition is not completely achieved $\left(\Omega_{2}-\Omega_{1} \gtrsim \kappa_{1}, \kappa_{2}\right)$, some of the oscillating field components in Eq. (A3) that were ignored should be taken into account: those oscillating at $\pm\left(\Omega_{2}-\Omega_{1}\right)$. Then the 
following terms are added to the right-hand side of the expressions for $b_{1}(\omega)$ and $b_{2}(\omega)$ in Eq. (A5):

$$
\begin{aligned}
b_{1}^{\prime}(\omega)= & \chi_{m, 1}(\omega) i \sum_{i}\left[\left(g_{i 1} \alpha_{i 2-}^{*}\right) a_{i}(\omega-\Delta \Omega)+\right. \\
& \left.\left(g_{i 1} \alpha_{i 2+}\right) a_{i}^{\dagger}(\omega-\Delta \Omega)\right], \\
b_{2}^{\prime}(\omega)= & \chi_{m, 2}(\omega) i \sum_{i}\left[\left(g_{i 2} \alpha_{i 1-}^{*}\right) a_{i}(\omega+\Delta \Omega)+\right. \\
& \left.\left(g_{i 2} \alpha_{i 1+}\right) a_{i}^{\dagger}(\omega+\Delta \Omega)\right],
\end{aligned}
$$

where $\Delta \Omega=\left(\Omega_{1}+\delta_{1}\right)-\left(\Omega_{2}+\delta_{2}\right)$. Similarly, the following term is added to the right-hand side of the expression for $a_{i}(\omega)$ in Eq. (A5):

$$
\begin{aligned}
a_{i}^{\prime}(\omega)= & \chi_{c, i}(\omega) i\left[\left(g_{i 1} \alpha_{i 2-}\right) b_{1}(\omega+\Delta \Omega)\right. \\
& +\left(g_{i 1} \alpha_{i 2+}\right) b_{1}^{\dagger}(\omega-\Delta \Omega) \\
& +\left(g_{i 2} \alpha_{i 1-}\right) b_{2}(\omega-\Delta \Omega) \\
& \left.+\left(g_{i 2} \alpha_{i 1+}\right) b_{2}^{\dagger}(\omega+\Delta \Omega)\right]
\end{aligned}
$$

Replacing the phononic operators in the expressions for photonic operators, we obtain $a_{i}(\omega)$ containing all $a_{k}(\omega), a_{k}^{\dagger}(\omega), a_{k}(\omega \pm \Delta \Omega), a_{k}^{\dagger}(\omega \pm \Delta \Omega), a_{k}(\omega \pm 2 \Delta \Omega)$, and $a_{k}^{\dagger}(\omega \pm 2 \Delta \Omega)$. We now determine what are the relevant generated terms if $\chi_{c, i}( \pm \Delta \Omega)$ is not ignored.

\section{First-order perturbation from interaction with off-resonant cavity photons}

Let us temporarily use the notation $A_{n}=\left(a_{1}(\omega+\right.$ $\left.n \Delta \Omega) a_{1}^{\dagger}(\omega+n \Delta \Omega) a_{2}(\omega+n \Delta \Omega) a_{2}^{\dagger}(\omega+n \Delta \Omega)\right)^{T}$ for $n \in \mathbb{Z}$, and similar notation for all other frequencydependent quantities. Under the rotating-wave approximation (RWA), the expression for $A_{n}$ involves a coupling matrix $T_{n}$, itself involving the mechanical susceptibility $\Xi_{m, n}$. Now that one of the RWAs is dropped, it involves many more coupling matrices containing mechanical susceptibilities $\Xi_{m, n}$ detuned by $n \Delta \Omega$, denoted $Q_{n}, R_{n}, S_{n}, V_{n}$, $W_{n}, X_{n}, Y_{n}$, and $Z_{n}$, representing different coupling mechanisms between cavity fields via mechanical oscillators, now that more of these mechanisms are driven. The expression for these matrices is determined below. Momentarily omitting any input signal or noise, we give the following result:

$$
\begin{aligned}
A_{n}= & -\Xi_{c, n}\left[Q_{n-1} A_{n-2}+\left(R_{n-1}+S_{n}\right) A_{n-1}\right. \\
& +\left(V_{n-1}+T_{n}+W_{n+1}\right) A_{n} \\
& +\left(X_{n}+Y_{n+1}\right) A_{n+1} \\
& \left.+Z_{n+1} A_{n+2}\right] .
\end{aligned}
$$

Since they all involve strong filtering by mechanical susceptibilities, all the coupling matrices can be ignored off resonance: $Q_{n}, R_{n}, S_{n}, V_{n}, W_{n}, X_{n}, Y_{n}, Z_{n}=0$ for $|n|>0$. Writing Eq. (C3) for $n=0$, one gets

$$
A_{0}=-\Xi_{c, 0}\left(T_{0} A_{0}+S_{0} A_{-1}+X_{0} A_{1}\right) .
$$

Inserting the expressions for $A_{ \pm 1}$, one obtains

$$
\begin{aligned}
A_{0}= & -\Xi_{c, 0}\left[\left(T_{0}+S_{0} \Xi_{c,-1} Y_{0}+X_{0} \Xi_{c, 1} R_{0}\right) A_{0}\right. \\
& +\left(S_{0} \Xi_{c,-1} W_{0}+X_{0} \Xi_{c, 1} Q_{0}\right) A_{-1} \\
& \left.+\left(S_{0} \Xi_{c,-1} Z_{0}+X_{0} \Xi_{c, 1} V_{0}\right) A_{1}\right] .
\end{aligned}
$$

All terms generated by the replacement of $A_{ \pm 1}$ in this new expression will contain products of off-resonant cavity susceptibilities such as $\boldsymbol{\Xi}_{c, \pm 1} \boldsymbol{\Xi}_{c, \pm 1}$. They are therefore ignored as higher-order terms. The development is then truncated at

$$
A_{0} \simeq-\Xi_{c, 0}\left(T_{0}+S_{0} \Xi_{c,-1} Y_{0}+X_{0} \Xi_{c, 1} R_{0}\right) A_{0} .
$$

To this order of development the coupling matrix around the resonance is modified by two terms representing interactions assisted by cavity photons from other manifolds. These two coefficients count because, for example, fields $a_{j}(\omega)$ involving $b_{j}(\omega+\Delta \Omega)$-and therefore $\chi_{m, j}(\omega+\Delta \Omega)$-were initially invoked at the frequency $\omega-\Delta \Omega$ and finally contributed with $\chi_{m, j}(\omega)$, which is non-negligible at $\omega \simeq 0$. In other words, trips on other frequency manifolds $\omega \simeq \pm \Delta \Omega$ are allowed provided excitations come back from them to the $\omega \simeq 0$ manifold.

\section{Explicit expressions of additional terms}

Equations $(\mathrm{C} 1)$ and $(\mathrm{C} 2)$ lead to the matrix form:

$$
\begin{aligned}
& A_{n}=\Xi_{c, n}\left(\mathcal{G} B_{n}+\mathcal{G}^{-} B_{n-1}+\mathcal{G}^{+} B_{n+1}\right), \\
& B_{n}=\Xi_{m, n}\left(\mathcal{H} A_{n}+\mathcal{H}^{-} A_{n-1}+\mathcal{H}^{+} A_{n+1}\right),
\end{aligned}
$$

with new coupling matrices $\mathcal{G}^{ \pm}$and $\mathcal{H}^{ \pm}$that couple photons or phonons to the previous or next manifold of phonons or photons. We introduce $\tilde{G}_{i j}\left(\tilde{J}_{i j}\right)$, the multiphoton optomechanical coupling of $\mathrm{MO} j$ to cavity $i$ enhanced by the red (blue) sideband intended for the MO other than $j$ (e.g., $\tilde{G}_{11}=g_{11} \alpha_{12-}$ ). The expressions for the additional coupling matrices are

$$
\begin{gathered}
\mathcal{G}^{-} \equiv-i\left(\begin{array}{cccc}
0 & \tilde{J}_{11} & \tilde{G}_{12} & 0 \\
0 & -\tilde{G}_{11}^{*} & -\tilde{J}_{12}^{*} & 0 \\
0 & \tilde{J}_{21} & \tilde{G}_{22} & 0 \\
0 & -\tilde{G}_{21}^{*} & -\tilde{J}_{22}^{*} & 0
\end{array}\right), \\
\mathcal{G}^{+} \equiv-i\left(\begin{array}{cccc}
\tilde{G}_{11} & 0 & 0 & \tilde{J}_{12} \\
-\tilde{J}_{11}^{*} & 0 & 0 & -\tilde{G}_{12}^{*} \\
\tilde{G}_{21} & 0 & 0 & \tilde{J}_{22} \\
-\tilde{J}_{21}^{*} & 0 & 0 & -\tilde{G}_{22}^{*}
\end{array}\right),
\end{gathered}
$$




$$
\begin{gathered}
\mathcal{H}^{-} \equiv-i\left(\begin{array}{cccc}
\tilde{G}_{11}^{*} & \tilde{J}_{11} & \tilde{G}_{21}^{*} & \tilde{J}_{21} \\
0 & 0 & 0 & 0 \\
0 & 0 & 0 & 0 \\
-\tilde{J}_{12}^{*} & -\tilde{G}_{12} & -\tilde{J}_{22}^{*} & -\tilde{G}_{22}
\end{array}\right), \\
\mathcal{H}^{+} \equiv-i\left(\begin{array}{cccc}
0 & 0 & 0 & 0 \\
-\tilde{J}_{11}^{*} & -\tilde{G}_{11} & -\tilde{J}_{21}^{*} & -\tilde{G}_{21} \\
\tilde{G}_{12}^{*} & \tilde{J}_{12} & \tilde{G}_{22}^{*} & \tilde{J}_{22} \\
0 & 0 & 0 & 0
\end{array}\right) .
\end{gathered}
$$

Replacing the phononic operators by their expression in the expression for the photonic operators, one identifies the expressions for coefficients $Q_{0}, R_{0}, S_{0}, V_{0}, W_{0}, X_{0}, Y_{0}$, and $Z_{0}$. Among these, the interesting ones according to Eq. (C6) are

$$
\begin{aligned}
S_{0}=-\mathcal{G} \Xi_{m}(\omega) \mathcal{H}^{-}, & Y_{0}=-\mathcal{G}^{+} \Xi_{m}(\omega) \mathcal{H}, \\
X_{0}=-\mathcal{G} \Xi_{m}(\omega) \mathcal{H}^{+}, & R_{0}=-\mathcal{G}^{-} \Xi_{m}(\omega) \mathcal{H},
\end{aligned}
$$

Both additional terms, $S_{0} \Xi_{c,-1} Y_{0}$ and $X_{0} \Xi_{c, 1} R_{0}$, in the coupling matrix $T$ have the same symmetry and the same zero elements as $T$; therefore, the phase-conjugating nature of the frequency conversion is not modified when this first-order perturbation is taken into account.

\section{Application to the case of the directional amplifier}

In the case of the directional amplifier studied here, the self-coupling coefficient $T_{11}(\omega)$ has two terms in the RWA framework:

$$
T_{11}(\omega)=\frac{C_{1} \kappa_{1}}{4}\left[\gamma_{1} \chi_{m, 1}(\omega)+\gamma_{2} \chi_{m, 2}(\omega)\right],
$$

which can be represented by the graphs in Fig. 7 .

The first-order perturbation adds the following terms to $T_{11}(\omega)$ :

$$
\begin{aligned}
T_{11}(\omega) \rightarrow & T_{11}(\omega) \\
& +\chi_{m, 1}^{2}(\omega)\left[\left|G_{11} \tilde{G}_{11}\right|^{2} \chi_{c, 1}(\omega-\Delta \Omega)\right. \\
& \left.-\left|G_{11} \tilde{J}_{21}\right|^{2} \chi_{c, 2}^{*}(\omega-\Delta \Omega)\right] \\
& +\chi_{m, 2}^{2}(\omega)\left[\left|G_{12} \tilde{G}_{12}\right|^{2} \chi_{c, 1}(\omega+\Delta \Omega)\right. \\
& \left.\quad-\left|G_{12} \tilde{J}_{22}\right|^{2} \chi_{c, 2}^{*}(\omega+\Delta \Omega)\right],(\mathrm{C} 14)
\end{aligned}
$$
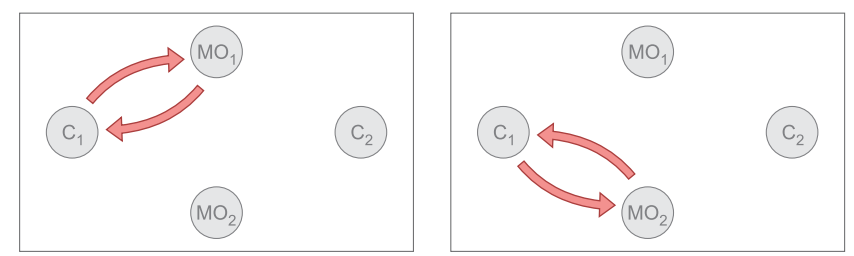

FIG. 7. RWA contributions to $T_{11} . \mathrm{C}_{1}$, cavity $1 ; \mathrm{C}_{2}$, cavity 2 ; $\mathrm{MO}_{1}, \mathrm{MO} 1 ; \mathrm{MO}_{2}, \mathrm{MO} 2$.

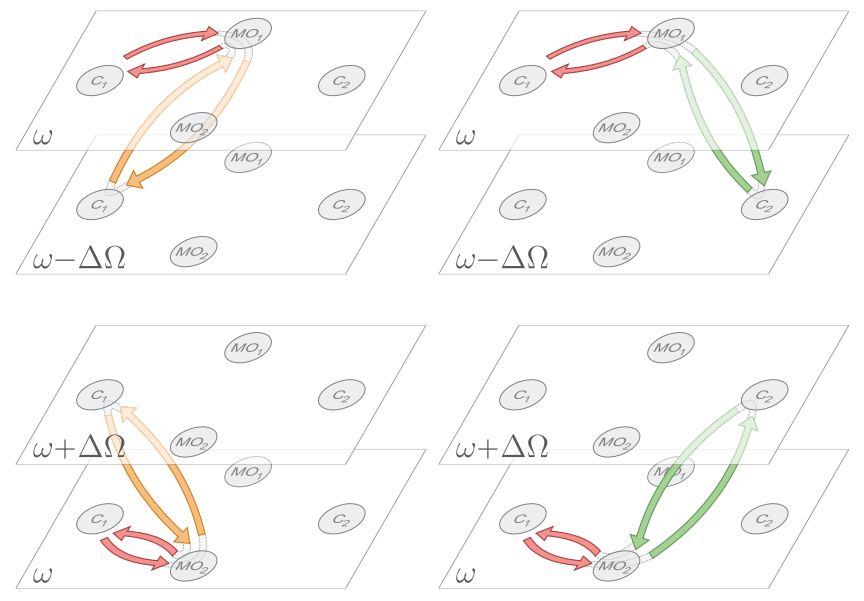

FIG. 8. Perturbations to $T_{11}$ to first order in $\kappa / \Delta \Omega$. $\mathrm{C}_{1}$, cavity $1 ; \mathrm{C}_{2}$, cavity $2 ; \mathrm{MO}_{1}, \mathrm{MO} 1 ; \mathrm{MO}_{2}, \mathrm{MO} 2$.

which are represented in several-manifold graphs as shown in Fig. 8.

These additional contributions are the result of the dynamical back-action due to the off-resonant terms from each cavity on each MO:

$$
\begin{array}{r}
\chi_{m, 1}(\omega) \rightarrow \chi_{m, 1}(\omega)\left[1+\chi_{m, 1}(\omega)\left|\tilde{G}_{11}\right|^{2} \chi_{c, 1}(\omega-\Delta \Omega)\right. \\
\left.-\chi_{m, 1}(\omega)\left|\tilde{J}_{21}\right|^{2} \chi_{c, 2}^{*}(\omega-\Delta \Omega)\right] \\
\chi_{m, 2}(\omega) \rightarrow \chi_{m, 1}(\omega)\left[1+\chi_{m, 2}(\omega)\left|\tilde{G}_{12}\right|^{2} \chi_{c, 1}(\omega+\Delta \Omega)\right. \\
\left.-\chi_{m, 2}(\omega)\left|\tilde{J}_{22}\right|^{2} \chi_{c, 2}^{*}(\omega+\Delta \Omega)\right] .
\end{array}
$$

In the RWA framework, the device's behavior is governed by the bare mechanical susceptibilities; that is, it is insensitive to back-action from the pump tones that drive each MO. Only the back-action due to tones not intended to drive each mode matters to the amplifier's quality (to first order in $\kappa / \Delta \Omega$ ). In terms of experimental parameters, the additional terms in $T_{11}$ are

$$
\begin{aligned}
& \gamma_{1} \gamma_{2} \chi_{m, 1}^{2}(\omega)\left[\left(\frac{g_{11}}{g_{12}}\right)^{2} \frac{C_{1}^{2} \kappa_{1}^{2}}{16} \chi_{c, 1}(\omega-\Delta \Omega)\right. \\
& \left.-\left(\frac{g_{21}}{g_{22}}\right)^{2} \frac{C_{2}^{2} \kappa_{2}^{2}}{16} \chi_{c, 2}^{*}(\omega-\Delta \Omega)\right] \\
& +\gamma_{1} \gamma_{2} \chi_{m, 2}^{2}(\omega)\left[\left(\frac{g_{12}}{g_{11}}\right)^{2} \frac{C_{1}^{2} \kappa_{1}^{2}}{16} \chi_{c, 1}(\omega+\Delta \Omega)\right. \\
& \left.-\left(\frac{g_{22}}{g_{21}}\right)^{2} \frac{C_{2}^{2} \kappa_{2}^{2}}{16} \chi_{c, 2}^{*}(\omega+\Delta \Omega)\right] .
\end{aligned}
$$



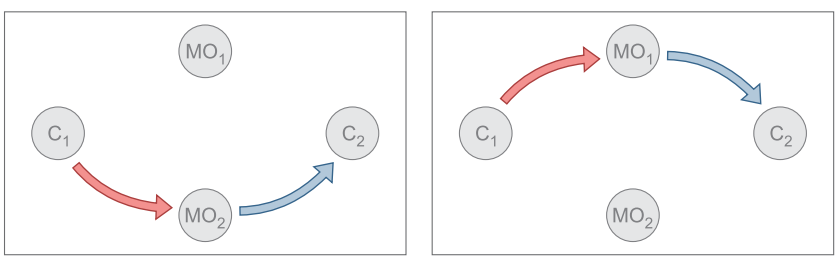

FIG. 9. RWA contributions to $T_{12} . \mathrm{C}_{1}$, cavity $1 ; \mathrm{C}_{2}$, cavity 2 ; $\mathrm{MO}_{1}, \mathrm{MO} 1 ; \mathrm{MO}_{2}, \mathrm{MO} 2$.

With the same idea, the zero-order expression for $T_{12}(\omega)$ involves two terms (made to interfere destructively)

$T_{12}(\omega)=\frac{\sqrt{C_{1} C_{2} \kappa_{1} \kappa_{2}}}{4}\left[e^{i \Phi / 2} \gamma_{1} \chi_{m, 1}(\omega)+e^{-i \Phi / 2} \gamma_{2} \chi_{m, 2}(\omega)\right]$,

which can be represented as in Fig. 9.

The first-order perturbation adds the terms

$$
\begin{aligned}
T_{12}(\omega) \rightarrow & T_{12}(\omega) \\
+ & +\chi_{m, 1}^{2}(\omega)\left[\left|\tilde{G}_{11}\right|^{2} G_{11} J_{21} \chi_{c, 1}(\omega-\Delta \Omega)\right. \\
& \left.\quad-\left|\tilde{J}_{21}\right|^{2} G_{11} J_{21} \chi_{c, 2}^{*}[\omega-\Delta \Omega)\right] \\
+ & \chi_{m, 2}^{2}(\omega)\left[\left|\tilde{G}_{12}\right|^{2} G_{12} J_{22} \chi_{c, 1}(\omega+\Delta \Omega)\right. \\
& \left.\quad-\left|\tilde{J}_{22}\right|^{2} G_{12} J_{22} \chi_{c, 2}^{*}(\omega+\Delta \Omega)\right],
\end{aligned}
$$

which are shown in a graph representation in Fig. 10.

In terms of experimental parameters, the additional terms are

$$
\begin{aligned}
& \frac{\gamma_{1} \gamma_{2} \sqrt{C_{1} C_{2} \kappa_{2} \kappa_{1}}}{16} \\
& \times\left\{\chi _ { m , 1 } ^ { 2 } ( \omega ) e ^ { i \Phi / 2 } \left[\left(\frac{g_{11}}{g_{12}}\right)^{2} \sqrt{C_{1} \kappa_{1}} \chi_{c, 1}(\omega-\Delta \Omega)\right.\right. \\
& \left.-\left(\frac{g_{21}}{g_{22}}\right)^{2} \sqrt{C_{2} \kappa_{2}} \chi_{c, 2}^{*}(\omega-\Delta \Omega)\right] \\
& +\chi_{m, 2}^{2}(\omega) e^{-i \Phi / 2}\left[\left(\frac{g_{12}}{g_{11}}\right)^{2} \sqrt{C_{1} \kappa_{1}} \chi_{c, 1}(\omega+\Delta \Omega)\right. \\
& \left.\left.-\left(\frac{g_{22}}{g_{21}}\right)^{2} \sqrt{C_{2} \kappa_{2}} \chi_{c, 2}^{*}(\omega+\Delta \Omega)\right]\right\} .
\end{aligned}
$$

Notably, only the phase $\Phi$ appears in these terms. Furthermore, the off-resonant cavity susceptibilities have real parts of different signs for back-action on MO 1 and MO 2: pump conditions calibrated for the RWA-framework terms to interfere destructively will not result in destructive interference of the terms developed in this section.
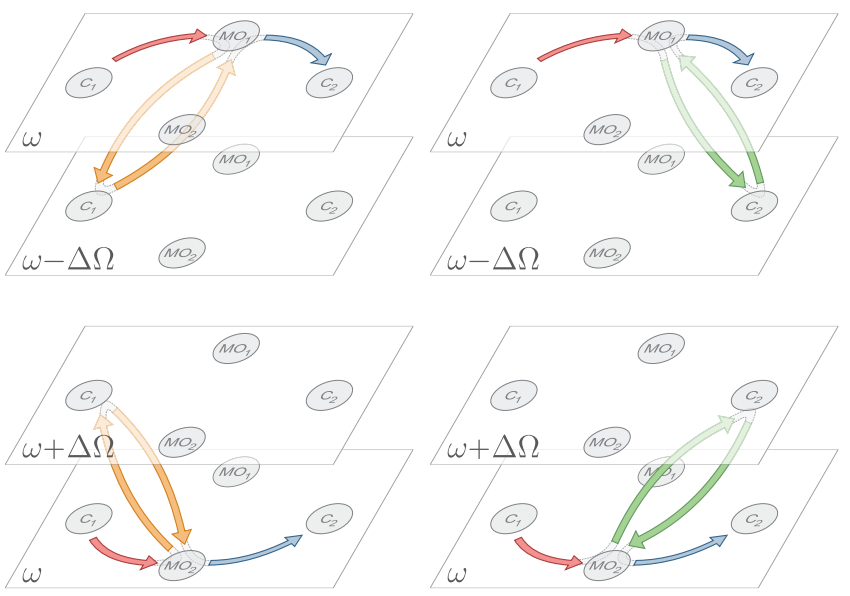

FIG. 10. Perturbations to $T_{12}$ to first order in $\kappa / \Delta \Omega$. $\mathrm{C}_{1}$, cavity $1 ; \mathrm{C}_{2}$, cavity $2 ; \mathrm{MO}_{1}, \mathrm{MO} 1 ; \mathrm{MO}_{2}, \mathrm{MO} 2$.

As a summary of the effect of these additional terms, we show the following:

(a) Each pump tone does not only address one mechanical mode but also induces a dynamical back-action on the second mechanical mode, which has consequences for the device (unlike the back-action of tones present within the RWA picture, which has no effect on the amplifier). The exact form of the effect is strongly dependent on the ratio of single-photon couplings, and generally influences the working point and bandwidth.

(b) The phase-insensitive (phase-conjugating) nature of the device is conserved.

(c) Only one pump phase appears as in the RWA picture, so, for example, there is no way to cancel out parasitic effects by tuning additional phase degrees of freedom.

(d) The ideal working point of the device will be modified and its quality may be altered since first-order terms do not interfere in the same way as zero-order terms. However, the additional dynamical back-action may happen to increase the device bandwidth if the modified mechanical resonances are broadened. This was observed in Ref. [37], where only red sidebands were driven, but is not necessarily the case here since the modes are subjected to back-action of different signs from the red and blue sidebands.

\section{APPENDIX D: NOISE SUBTRACTION}

The network analyzer measures the ratio of input power to output power $S_{i j \text {,meas }}(\omega)=P_{\mathrm{in}, i}(\omega) / P_{\text {out } j}$ where $i$ and $j$ denote the frequency ranges around cavities $i$ and $j$ (note that here input and output denominations are chosen with respect to the measurement instrument and not the device). Along with the power obtained from the amplifier excitation, the power corresponding to noise integration on the network analyzer's bandwidth $B(B=20 \mathrm{~Hz})$ also con- 
tributes to $P_{\mathrm{in}, i}$. The noise is therefore independently measured with a spectrum analyzer that gives the power $P_{\text {noise }}$ integrated on the spectrum analyzer's resolution bandwidth $R(R=30 \mathrm{~Hz})$. Subsequently this noise is removed from the measured $S$ parameters, taking into account the different integration bandwidths, to yield the pure transmission coefficients:

$$
S_{i j}(\omega)=S_{i j, \text { meas }}(\omega)-\frac{B}{R} \frac{P_{\text {noise }, i}(\omega)}{P_{\text {out }, j}} .
$$

\section{APPENDIX E: GAIN CALIBRATION}

After noise subtraction, the transfer parameters of the amplifier are extracted from the measured signals; that is, these signals are corrected by a factor taking into account the total attenuation and amplification from cables, amplifiers, and other elements on the input and output lines of the cryostat.

The input and output sides of these measurement lines show a frequency dependence. The four transfer parameters are measured by pumping and probing very different frequency ranges around the two resonances of the superconducting circuit (5.6 and $3.9 \mathrm{GHz}$ ); therefore, they are affected by different gains. More precisely, the raw measured transfer coefficients are related to the device transfer coefficients by

$$
\begin{aligned}
& S_{11}^{\text {raw }}=\eta_{1}^{\text {in }} \eta_{1}^{\text {out }} S_{11}, \\
& S_{12}^{\text {raw }}=\sqrt{\omega_{1} / \omega_{2}} \eta_{2}^{\text {in }} \eta_{1}^{\text {out }} S_{12}, \\
& S_{21}^{\text {raw }}=\sqrt{\omega_{2} / \omega_{1}} \eta_{1}^{\text {in }} \eta_{2}^{\text {out }} S_{21}, \\
& S_{22}^{\text {raw }}=\eta_{2}^{\text {in }} \eta_{2}^{\text {out }} S_{22},
\end{aligned}
$$

where $\eta^{\text {in }}$ are pump efficiencies characterizing the total attenuation on the input side, $\eta^{\text {out }}$ are measurement efficiencies denoting the total amplification on the output side, and indices 1 and 2 represent the two ranges of frequencies (around cavity 1 and cavity 2). The factor $\sqrt{\omega_{2} / \omega_{1}}$ and its inverse scale the transfer parameters measured in terms of power by the network analyzer into parameters in terms of photon numbers when the frequencies of the probe and pump photons are not the same. These gains are assumed not to vary significantly in each of these frequency ranges, which seems a very reasonable assumption from their typical frequency dependence out of cavity resonance.

The two measurement system gains affecting $S_{11}$ and $S_{22}$ are easily measured from the response of the device out of, but close to, the cavity resonances, as explained in the main text: $20 \log _{10}\left(\eta_{1}^{\text {in }} \eta_{1}^{\text {out }}\right)=-61.2 \pm 0.1 \mathrm{~dB}$ and $20 \log _{10}\left(\eta_{2}^{\text {in }} \eta_{2}^{\text {out }}\right)=-58.8 \pm 0.2 \mathrm{~dB}$. The uncertainties represent the typical standard deviations of $S_{11}$ and $S_{22}$ out of resonance.
However, the two other gains, which affect $S_{12}$ and $S_{21}$, cannot be estimated in the same way since there is no frequency-converting transfer out of cavity resonances. The product of these transmission gains is, however, known (equal to the product of the two reflection gains):

$$
20 \log _{10}\left(\eta_{2}^{\text {in }} \eta_{1}^{\text {out }} \times \eta_{1}^{\text {in }} \eta_{2}^{\text {out }}\right)=-120.0 \pm 0.3 \mathrm{~dB}
$$

Single-mechanical-oscillator amplifiers are reciprocal $S_{12}=S_{21}$ [40]. The raw transmissions of both amplifiers built with each of the mechanical oscillators, whose measurement is presented in Fig. 11, satisfy

$$
\begin{aligned}
20 \log _{10}\left(\left|S_{12}^{\text {raw }} / S_{21}^{\text {raw }}\right|\right) & =20 \log _{10}\left(\eta_{2}^{\text {in }} \eta_{1}^{\text {out }} \omega_{1} / \eta_{1}^{\text {in }} \eta_{2}^{\text {out }} \omega_{2}\right) \\
& =10.0 \pm 0.8 \mathrm{~dB} .
\end{aligned}
$$

The uncertainty corresponds to the sum of the standard deviations of $S_{12}$ and $S_{21}$ data for single-MO amplifiers from which the fit value was removed. Because of the reciprocity of each amplifier, apart from the frequencyratio factor, this imbalance accounts only for the difference between the products $\eta_{2}^{\text {in }} \eta_{1}^{\text {out }}$ and $\eta_{1}^{\text {in }} \eta_{2}^{\text {out }}$. The remaining
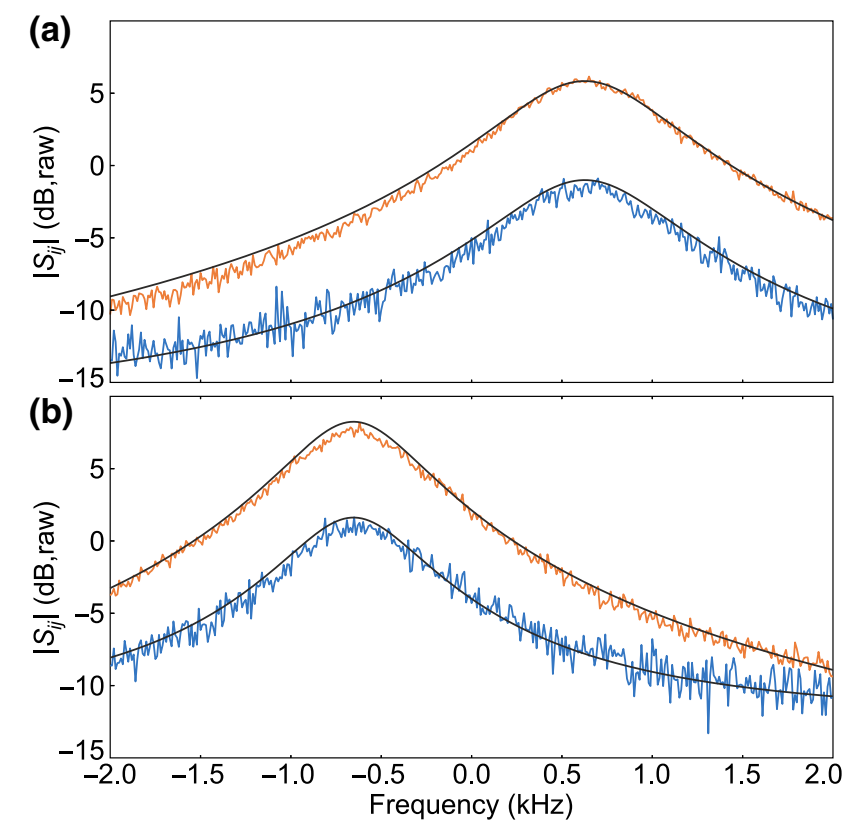

FIG. 11. Single-MO amplifiers used for cross-gain calibration. (a) Parameters $\left|S_{12}\right|$ (orange) and $\left|S_{21}\right|$ (blue) of the singleMO amplifier built with MO 1, corrected by the average gain $\sqrt{\eta_{2}^{\text {in }} \eta_{1}^{\text {out }} \eta_{1}^{\text {in }} \eta_{2}^{\text {out }}}$, still showing an imbalance of $6.8 \mathrm{~dB}$. This value is calibrated from the fits in black lines. A constant background is added in the fit expression to reproduce the noise floor. (b) Same data taken for the other single-MO amplifier built with MO 2, showing the same imbalance of $6.8 \mathrm{~dB}$, obtained from a separate fit. 
gains can be computed from this imbalance:

$$
\begin{aligned}
& 20 \log _{10}\left(\eta_{2}^{\text {in }} \eta_{1}^{\text {out }}\right)=-56.6 \pm 1.1 \mathrm{~dB}, \\
& 20 \log _{10}\left(\eta_{1}^{\text {in }} \eta_{2}^{\text {out }}\right)=-63.4 \pm 1.1 \mathrm{~dB} .
\end{aligned}
$$

This calibration entails an input efficiency difference of $20 \log _{10}\left(\eta_{1}^{\text {in }} / \eta_{2}^{\text {in }}\right)=-4.6 \mathrm{~dB}$ between the frequency ranges of cavity $1(5.6 \mathrm{GHz})$ and cavity $2(3.9 \mathrm{GHz})$. This is probably due to the use of resistive lines on this side of the measurement system, which display higher losses at higher frequencies. On the other hand, the gain discrepancy has the other sign on the output side: $20 \log _{10}\left(\eta_{1}^{\text {out }} / \eta_{2}^{\text {out }}\right)=$ $2.2 \mathrm{~dB}$. An independent calibration of the output lines confirms the $2.2 \pm 0.3 \mathrm{~dB}$ lower gain at the lower frequency of cavity $2(3.9 \mathrm{GHz})$ compared with cavity $1(5.6 \mathrm{GHz})$, mainly due to the proximity to the cut frequency of a high-pass filter $(4 \mathrm{GHz})$.

[1] M. Hafezi, and P. Rabl, Optomechanically induced nonreciprocity in microring resonators, Opt. Express 20, 7672 (2012).

[2] Z. Shen, Y.-L. Zhang, Y. Chen, C.-L. Zou, Y.-F. Xiao, X.-B. Zou, F.-W. Sun, G.-C. Guo, and C.-H. Dong, Experimental realization of optomechanically induced non-reciprocity, Nat. Photonics 10, 657 (2016).

[3] F. Ruesink, M.-A. Miri, A. Alú, and E. Verhagen, Nonreciprocity and magnetic-free isolation based on optomechanical interactions, Nat. Commun. 7, 13662 (2016).

[4] Z. Shen, Y.-L. Zhang, Y. Chen, F.-W. Sun, X.-B. Zou, G.-C. Guo, C.-L. Zou, and C.-H. Dong, Reconfigurable optomechanical circulator and directional amplifier, Nat. Commun. 9, 1797 (2018).

[5] F. Ruesink, J. P. Mathew, M.-A. Miri, A. Alù, and E. Verhagen, Optical circulation in a multimode optomechanical resonator, Nat. Commun. 9, 1798 (2018).

[6] B. Abdo, K. Sliwa, L. Frunzio, and M. Devoret, Directional Amplification with a Josephson Circuit, Phys. Rev. X 3, 031001 (2013).

[7] B. Abdo, K. Sliwa, S. Shankar, M. Hatridge, L. Frunzio, R. Schoelkopf, and M. Devoret, Josephson Directional Amplifier for Quantum Measurement of Superconducting Circuits, Phys. Rev. Lett. 112, 167701 (2014).

[8] A. Kamal, A. Roy, J. Clarke, and M. H. Devoret, Asymmetric Frequency Conversion in Nonlinear Systems Driven by a Biharmonic Pump, Phys. Rev. Lett. 113, 247003 (2014).

[9] K. M. Sliwa, M. Hatridge, A. Narla, S. Shankar, L. Frunzio, R. J. Schoelkopf, and M. H. Devoret, Reconfigurable Josephson Circulator/Directional Amplifier, Phys. Rev. X 5, 041020 (2015).

[10] F. Lecocq, L. Ranzani, G. A. Peterson, K. Cicak, R. W. Simmonds, J. D. Teufel, and J. Aumentado, Nonreciprocal Microwave Signal Processing with a Field-Programmable Josephson Amplifier, Phys. Rev. Appl. 7, 024028 (2017).
[11] M. P. Westig, and T. M. Klapwijk, Josephson Parametric Reflection Amplifier with Integrated Directionality, Phys. Rev. Appl. 9, 064010 (2018).

[12] M. Soljačić, C. Luo, J. D. Joannopoulos, and S. Fan, Nonlinear photonic crystal microdevices for optical integration, Optics Lett. 28, 637 (2003).

[13] L. Fan, J. Wang, L. T. Varghese, H. Shen, B. Niu, Y. Xuan, A. M. Weiner, and M. Qi, An all-silicon passive optical diode, Science 335, 447 (2012).

[14] D. L. Sounas, and A. Alù, Angular-momentum-biased nanorings to realize magnetic-free integrated optical isolation, ACS. Photonics. 1, 198 (2014).

[15] Z. Wu, J. Chen, M. Ji, Q. Huang, J. Xia, Y. Wu, and Y. Wang, Optical nonreciprocal transmission in an asymmetric silicon photonic crystal structure, Appl. Phys. Lett. 107, 221102 (2015).

[16] X. Guo, C.-L. Zou, H. Jung, and H. X. Tang, OnChip Strong Coupling and Efficient Frequency Conversion between Telecom and Visible Optical Modes, Phys. Rev. Lett. 117, 123902 (2016).

[17] S. Hua, J. Wen, X. Jiang, Q. Hua, L. Jiang, and M. Xiao, Demonstration of a chip-based optical isolator with parametric amplification, Nat. Commun. 7, 13657 (2016).

[18] Z. Yu, and S. Fan, Complete optical isolation created by indirect interband photonic transitions, Nat. Photonics 3, 91 (2009).

[19] H. Lira, Z. Yu, S. Fan, and M. Lipson, Electrically Driven Nonreciprocity Induced by Interband Photonic Transition on a Silicon Chip, Phys. Rev. Lett. 109, 033901 (2012).

[20] N. A. Estep, D. L. Sounas, J. Soric, and A. Alù, Magneticfree non-reciprocity and isolation based on parametrically modulated coupled-resonator loops, Nat. Phys. 10, 923 (2014).

[21] F. Yang, and Y. Li, Nonreciprocal diffraction of light based on double-transition-assisted photonic AharonovBohm effect, Phys. Rev. B 94, 165439 (2016).

[22] Y. Jiang, S. Maayani, T. Carmon, F. Nori, and H. Jing, Nonreciprocal Phonon Laser, Phys. Rev. Appl. 10, 064037 (2018).

[23] L. Ranzani, and J. Aumentado, Graph-based analysis of nonreciprocity in coupled-mode systems, New J. Phys. 17, 023024 (2015).

[24] A. Metelmann, and A. A. Clerk, Nonreciprocal Photon Transmission and Amplification via Reservoir Engineering, Phys. Rev. X 5, 021025 (2015).

[25] K. Fang, J. Luo, A. Metelmann, M. H. Matheny, F. Marquardt, A. A. Clerk, and O. Painter, Generalized non-reciprocity in an optomechanical circuit via synthetic magnetism and reservoir engineering, Nat. Phys. 13, 465 (2017).

[26] Y. Li, Y. Y. Huang, X. Z. Zhang, and L. Tian, Optical directional amplification in a three-mode optomechanical system, Opt. Express 25, 18907 (2017).

[27] L. Tian, and Z. Li, Nonreciprocal quantum-state conversion between microwave and optical photons, Phys. Rev. A 96, 013808 (2017).

[28] C. Jiang, L. N. Song, and Y. Li, Directional amplifier in an optomechanical system with optical gain, Phys. Rev. A 97, 053812 (2018). 
[29] G. Li, X. Xiao, Y. Li, and X. Wang, Tunable optical nonreciprocity and a phonon-photon router in an optomechanical system with coupled mechanical and optical modes, Phys. Rev. A 97, 023801 (2018).

[30] H. Xu, L. Jiang, A. A. Clerk, and J. G. E. Harris, Nonreciprocal control and cooling of phonon modes in an optomechanical system, arXiv:1807.03484.

[31] I. Moaddel Haghighi, N. Malossi, R. Natali, G. Di Giuseppe, and D. Vitali, Sensitivity-Bandwidth Limit in a Multimode Optoelectromechanical Transducer, Phys. Rev. Appl. 9, 034031 (2018).

[32] B. H. Eom, P. K. Day, H. G. LeDuc, and J. Zmuidzinas, A wideband, low-noise superconducting amplifier with high dynamic range, Nature Physics 8, 623 (2012).

[33] C. Macklin, K. O’Brien, D. Hover, M. E. Schwartz, V. Bolkhovsky, X. Zhang, W. D. Oliver, and I. Siddiqi, A near-quantum-limited Josephson traveling-wave parametric amplifier, Science 350, 307 (2015).

[34] X.-W. Xu, and Y. Li, Optical nonreciprocity and optomechanical circulator in three-mode optomechanical systems, Phys. Rev. A 91, 053854 (2015).

[35] X.-W. Xu, Y. Li, A.-X. Chen, and Y.-X. Liu, Nonreciprocal conversion between microwave and optical photons in electro-optomechanical systems, Phys. Rev. A 93, 023827 (2016).

[36] G. A. Peterson, F. Lecocq, K. Cicak, R. W. Simmonds, J. Aumentado, and J. D. Teufel, Demonstration of Efficient Nonreciprocity in a Microwave Optomechanical Circuit, Phys. Rev. X 7, 031001 (2017).

[37] N. R. Bernier, L. D. Tóth, A. Koottandavida, M. A. Ioannou, D. Malz, A. Nunnenkamp, A. K. Feofanov, and T. J. Kippenberg, Nonreciprocal reconfigurable microwave optomechanical circuit, Nat. Commun. 8, 604 (2017).

[38] S. Barzanjeh, M. Wulf, M. Peruzzo, M. Kalaee, P. B. Dieterle, O. Painter, and J. M. Fink, Mechanical on-chip microwave circulator, Nat. Commun. 8, 953 (2017).

[39] F. Massel, T. T. Heikkilä, J. M. Pirkkalainen, S. U. Cho, H. Saloniemi, P. J. Hakonen, and M. A. Sillanpää, Microwave amplification with nanomechanical resonators, Nature $\mathbf{4 8 0}$, 351 (2011).

[40] C. F. Ockeloen-Korppi, E. Damskägg, J.-M. Pirkkalainen, T. T. Heikkilä, F. Massel, and M. A. Sillanpää, Low-noise amplification and Frequency Conversion with a Multiport Microwave Optomechanical Device, Phys. Rev. X 6, 041024 (2016).

[41] L. D. Tóth, N. R. Bernier, A. Nunnenkamp, A. K. Feofanov, and T. J. Kippenberg, A dissipative quantum reservoir for microwave light using a mechanical oscillator, Nat. Phys. 13, 787 (2017).

[42] C. F. Ockeloen-Korppi, E. Damskägg, J.-M. Pirkkalainen, T. T. Heikkilä, F. Massel, M. A. Sillanpää, Noiseless Quantum Measurement and Squeezing of Microwave Fields Utilizing Mechanical Vibrations, Phys. Rev. Lett. 118, 103601 (2017).

[43] D. Malz, L. D. Tóth, N. R. Bernier, A. K. Feofanov, T. J. Kippenberg, and A. Nunnenkamp, Quantum-Limited Directional Amplifiers with Optomechanics, Phys. Rev. Lett. 120, 023601 (2018).

[44] C. M. Caves, Quantum limits on noise in linear amplifiers, Phys. Rev. D 26, 1817 (1982).

[45] C. F. Ockeloen-Korppi, T. T. Heikkilä, M. A. Sillanpää, and F. Massel, Theory of phase-mixing amplification in an optomechanical system, Quantum Sci. Technol. 2, 035002 (2017).

[46] J. D. Teufel, T. Donner, D. Li, J. W. Harlow, M. S. Allman, K. Cicak, A. J. Sirois, J. D. Whittaker, K. W. Lehnert, and R. W. Simmonds, Sideband cooling of micromechanical motion to the quantum ground state, Nature 475, 359 (2011). 